\title{
Transcriptional condensates formed by phase-separated ALOG family proteins control shoot meristem maturation for flowering
}

Xiaozhen Huang ${ }^{1,3,5}$, Nan Xiao ${ }^{1,2,3,5}$, Yue Xie ${ }^{1,2,3}$, Lingli Tang ${ }^{1,2,3}$, Yueqin Zhang ${ }^{1,3,4}$, Yuan Yu $u^{1,2,3}$, and Cao $\mathrm{Xu}^{1,3, *}$

${ }^{1}$ State Key Laboratory of Plant Genomics, National Center for Plant Gene Research (Beijing), Institute of Genetics and Developmental Biology, The Innovative Academy of Seed Design, Chinese Academy of Sciences, Beijing, China.

${ }^{2}$ University of Chinese Academy of Sciences, Beijing, China.

${ }^{3}$ CAS-JIC Centre of Excellence for Plant and Microbial Science (CEPAMS), Institute of Genetics and Developmental Biology, Chinese Academy of Sciences, Beijing, China.

${ }^{4}$ College of Coastal Agricultural Sciences Guangdong Ocean University, Zhanjiang, China.

${ }^{5}$ These authors contributed equally to this work.

*Address correspondence to caoxu@genetics.ac.cn. 


\section{Abstract}

Plants have evolved remarkable diversity in inflorescence architecture. At the center of this diversity lies a meristem maturation program featured by transition of stem cell populations from a vegetative state into a reproductive growth, determining when, where, and how many flowers are produced on inflorescences. Here we identified a new meristem maturation regulator TMF FAMILY MEMBER3 (TFAM3) that encodes an ALOG family transcription factor. Loss of TFAM3 results in early flowering and simplified inflorescences with fewer flowers. Genetic analysis by creating high-order mutants of TFAM3 with three key regulators of tomato shoot meristem maturation, TERMINATING FLOWER (TMF), TMF FAMILY MEMBER1 (TFAM1) and TMF FAMILY MEMBER2 (TFAM2), suggested that they synergistically control flowering transition and inflorescence architecture. The four paralogous ALOG proteins share the prion-like properties and undergo liquid-liquid phase separation in vitro. Strikingly, TMF can recognize cognate TFAM proteins and selectively recruit them into phase separated condensates. Supporting this, they interact with themselves and each other to form biomolecular condensates in the nucleus. Their interaction induces formation of transcriptional condensates that directly repress expression of floral identity gene $A N A N T H A$. Our study revealed a selective-recruitment phase separation mechanism for transcriptional condensation by which plants achieve optimal coordination of functional overlapped paralogs within a protein family to enable precise control of shoot meristem maturation for flowering and production of compound inflorescences.

\section{Introduction}

Reproductive success and shoot architectural diversity of flowering plants rely on the time of flowering and the architecture of inflorescences (Benlloch et al., 2007; Rickett, 1944; Weberling, 1989; Xu et al., 2016). Inflorescences develop from shoot apical meristems (SAMs), whose fate 
are determined by balancing cell proliferation for replenishing pools of pluripotent cells with cell differentiation for organogenesis (Huang et al., 2021). Upon perception and integration of endogenous and environmental signals, SAMs experience a gradual maturation process from a vegetative stage (vegetative meristem, VM) into a floral stage (floral meristem, FM) accompanied by successive leaf production, called meristem maturation (Park et al., 2012, 2014).

The programed and successive meristem maturation ensures the transition to flowering at proper time and the formation of flowers in appropriate amount, which directly influence reproductive success of plants and yield of crops (Park et al., 2014; Xu et al., 2016). Before reaching the transition stage, SAM at vegetative stage is too juvenile to respond to endogenous or environmental signals. A program that prevents precocious maturation is vital for enabling a sufficient duration of vegetative stage for accumulation of adequate stem cells. Upon "juvenile" meristem maturing to "adult" meristem, a promoting program is required to activate floral identity gene for flowering. Therefore, both repressing and promoting programs during meristem maturation are essential for precise control of flowering transition and inflorescence architecture.

Over the past decades, studies in model and crop plants have reported genetic pathways that implicate in flowering transition and the formation of diverse inflorescence architectures (Barton, 2010; González-Suárez et al., 2020; Teo et al., 2014; Wu et al., 2018; Zhu and Wagner, 2020). Studies on tomato inflorescence mutants and wild species have come up with a working model that illustrates the mechanism for promoting meristem maturation. Once the promoting program is disrupted, meristem maturation is delayed or fails to complete, giving rising to over-proliferated axillary inflorescence meristems (AIMs) and thus highly branched inflorescences (Park et al., 2012). Loss-of-function of homeobox gene COMPOUND INFLORESCENCE (S) in tomato or EVERGREEN (EVG) in petunia, both genes are homologs of Arabidopsis WOX9, leads to highly branched inflorescences (Hendelman et al., 2021; Lippman et al., 2008; Rebocho et al., 2008). Mutant of the F-box gene ANANTHA (AN; homolog of Arabidopsis UFO and petunia DOT) in tomato never forms normal inflorescences and flowers, only produces overproliferated axillary inflorescence meristems (Hepworth et al., 2006; Lippman et al., 2008; Rebocho et al., 2008; Souer 
et al., 2008). Genetic studies together with tissue-specific transcriptome profiling of SAMs in tomato cultivar and wild species support a model wherein programmed activation of $S$ followed by $A N$ drives successive stages of axillary inflorescence meristems maturation to ensure them develop at precise time and in appropriate amount in each sympodial growth cycle (Park et al., 2012, 2014).

A repressing program that maintains meristem at a vegetative state is defined by a tomato ALOG (Arabidopsis LSH1 and Oryza G1) transcription factor, TERMINATING FLOWER (TMF) (MacAlister et al., 2012). TMF harbors a conserved ALOG domain featured by a putative DNAbinding domain derived from the XerC/D-like recombinases of a novel class of retrotransposons (Iyer and Aravind, 2012; Xu et al., 2016). Loss of TMF in tomato leads to much faster flowering and conversion of the multiple-flowered primary inflorescence into a single flower. These effects caused by precocious activation of $A N$ due to loss of TMF's repression, making a meristem mostly at a vegetative stage acquire the floral identity (MacAlister et al., 2012). Surprisingly, inflorescences that develop from side shoots of $t m f$ mutant are normal, implying that $T M F$ paralogs in ALOG family or other genes might synergistically act with $T M F$ to regulate the inflorescence architecture. Indeed, the tomato ALOG family includes twelve members, which are named as TMF FAMILY MEMBERs (TFAMs) (Xu et al., 2016). Two additional ALOG family members, TFAM1 and TFAM2, form transcriptional complex with TMF via homo- and hetero-interactions to act together in preventing precocious meristem maturation (Huang et al., 2018). Studies in Arabidopsis and other nightshades (Solanaceae) have reported important roles of ALOG genes in floral organ specification, light signaling (Chen et al., 2019; Cho and Zambryski, 2011; Takeda et al., 2011; Zhao et al., 2004). In rice and wheat, several ALOG family members have been found to regulate lemma and hull specification, spikelet development, panicle branching and grain size (Nan et al., 2018; Sato et al., 2014; Yoshida et al., 2009, 2013). Notably, loss of Marchantia polymorpha LATERAL ORGAN SUPRESSOR1 (MpLOS1), a TMF ortholog in the liverwort, causes mis-specified identity of lateral organogenesis and defects in apical meristem maintenance, suggesting its essential role in convergent evolution of lateral organogenesis (Naramoto et al., 
2019). ALOG proteins represent a new plant specific transcription factor family with vital functions in meristem activity and reproductive development, however, the molecular mechanism of their transcriptional regulation is poorly understood.

The plant meristem maturation is a programmed developmental event driven by precise switch of gene repression and activation, which might require integration and transmission of endogenous and environmental signals into gene expression to be finely controlled in a relatively undisturbed micro-environment. Compartmentalization within a cell is a widely used strategy for complicated cellular processes and biological reactions in multicellular organisms. Besides canonical membrane-bound organelles, biomolecular condensates, the microscale membraneless compartments formed by liquid-liquid phase separation, have been recently found to spatially concentrate proteins and nucleic acids (Banani et al., 2017). To date biomolecular condensates formed by protein phase separation have recently been demonstrated as a new strategy for many cellular functions in yeast and animal systems, including cell polarity establishment and maintenance, cell signaling, cell and organ development, cell survival, and aging (Alberti and Hyman, 2021; Banani et al., 2017; Boeynaems et al., 2018; Strzyz, 2019; Wu et al., 2020). However, the mechanism of protein phase separation and the function of derived biomolecular condensates in plants are still largely uninvestigated (Emenecker et al., 2021; Fang et al., 2019; Huang et al., 2021; Zhang et al., 2020). In this study, we found that phase-separation induced formation of a transcriptional condensate times floral identity gene expression to program shoot apical meristem maturation in tomato.

\section{Results}

\section{$T F A M 3$ is a new regulator of flowering transition and inflorescence architecture}

Biological robustness is the ability of a system to maintain its function despite environmental or genetic perturbation (Diss et al., 2014). Genetic robustness can be achieved by functionally overlapped paralogs within a gene family. The phenomenon of this kind of redundancy is not rare, 
however, the molecular mechanisms linking the genotype to the phenotype are still poorly understood. The fact that TMF, TFAM1 and TFAM2 act synergistically in meristem maturation prompts us to systemically explore the roles of TMF family members in this process to uncover the mechanism underlying their paralogous interactions. Phylogeny analysis and protein alignment showed that twelve TFAM family members can be divided into two clades. Seven TFAMs, TMF and TFAM 1, 2, 3, 5, 9, 11, were clustered into one group, in which TFAM3 is the closest paralog of TMF (Figure 1A). To focus on the potential partners of TMF and TFAM1/2, we first examine the expression pattern of TFAMs in meristems at various developmental stages. The tissue-specific transcriptome profiling data suggested that four members in this clade, TMF and TFAM1, 2, 3, 11, show predominantly expression in meristems (Park et al., 2012). Among them, TFAM3 shows similarity to $T M F$ and TFAM1, 2, which express highly during VM and TM stages and then decrease in FM, but TFAM11 shows low expression in VMs and high expression in FM, being distinct from that of TMF (Figure 1B). In this regard, we use CRISPR/Cas9 system to knock out TFAM3 (Figure 1C). The tfam3 null mutant flowers about one leaf earlier and produces fewer flowers on the primary inflorescence compared with wild type (Figure 1D-F), resembling mutant phenotypes of TMF weak alleles (Huang et al., 2021; MacAlister et al., 2012). Moreover, about $13 \%$ of the primary inflorescences of $\operatorname{tfam} 3$ show vegetative reversion, indicated by outgrowth of leaves on the inflorescence (Figure 1D). The tfam3 mutant frequently underwent a single branching event on each inflorescence (Figure 1D). These phenotypes remind us the inflorescence development defects of tfam 1 and tfam 2 mutants. As previously reported and shown here, the flowering time is unaffected in tfam 1 and tfam 2 single mutants (Figure 1D and 1E). Instead, tfam 1 shows reduced flower production but highly frequent vegetative reversion on inflorescences, and tfam 2 develops inflorescences with a high frequency of single branching event (Figure 1D and 1F) (Xu et al., 2016). These findings suggest that TFAM3 is a novel regulator that represses flowering transition and promotes inflorescence complexity. The partially phenotypic similarity of the three tfam mutants suggests their functional overlap. 


\section{Higher-order tfam mutants show enhanced early-flowering and inflorescence defects}

To explore how TFAM3, TFAM1 and TFAM2 coordinate in synchronizing flowering transition and promoting inflorescence complexity, we generated various combinations of higher-order mutants by genetic crosses. Loss of either TFAM1 or TFAM2 in tfam 3 mutant background enhances its early flowering phenotypes, showing one leaf earlier than tfam 3 single mutant, about two leaves earlier than wild type plants (Figure 1D and 1E). The tfam 1/2 flowered faster than either single mutant by about one leaf, showing weaker early-flowering phenotype than tfam 1/3 and tfam 2/3 (Figure $1 \mathrm{D}$ and $1 \mathrm{E})$. In addition to enhanced flowering phenotypes, the tfam double mutants showed a range of modifications to inflorescence architecture. Most dramatically, tfam 1/3 inflorescence produces only less than half of the number of flowers that the wild-type plant does, and about 38\% inflorescences show vegetative reversion, similar to that of tfam 1 but stronger than that of tfam 3 (Figure 1D-1F). In contrast, tfam $2 / 3$ and $t$ fam $1 / 2$ only displayed a slight change of the frequency of vegetative reversion and branching in comparison with single mutants (Figure 1D-1F). Strikingly, comparing to tfam doubles, the tfam 1/2/3 triple mutant flowered even faster, and its inflorescences only produced about three flowers (Figure 1D-1F). The progressive enhancement of flowering and inflorescence defects displayed by a complete series of $t$ fam mutants indicate that their inseparable relationship in modulating flowering time and inflorescence architecture.

\section{$T M F$ acts together with $T F A M s$ to control flowering and inflorescence complexity}

The $T M F$ gene is a core member of ALOG family in tomato and plays pivotal role in regulating flowering and inflorescence complexity (MacAlister et al., 2012; Xu et al., 2016). The earlyflowering and simplified-inflorescence phenotypes of various tfam mutants are similar, albeit weaker, to tmf null mutant. We therefore hypothesize that TMF might function as a "leader" to recruit and organize three TFAMs. To address this, we crossed all the single and multiple tfam mutants with $\operatorname{tmf}$ to create a series of tmf tfam mutant combinations. Among the double mutants, tmf tfam 1 and tmf tfam 3 showed the most significant enhancement of early-flowering comparing to $\operatorname{tmf}$ (Figure 2A and 2B). In contrast, there is no significant difference in flowering between tmf 
tfam2 and tmf (Figure 2A and 2B). More prominent enhancement of early-flowering occurs in triple mutants. The tmf tfam 1/2 and tmftfam 1/3 flowered earlier than $t m f$ single mutant by one and two leaves, respectively (Figure 2A and 2B). Strikingly, the tmf tfam 1/2/3 quadruple mutant flowered extremely early after producing only two leaves and developed single-flowered inflorescences (Figure 2A and 2B). In addition, the $t m f$ single-flower phenotype showed about 80\% penetrance in our growth condition, neither introduction of TFAM1, 2 mutation individually nor simultaneously significantly improves the penetrance. However, the tmf tfam 1/2/3 quadruple mutant showed almost 100\% penetrance for the single-flower phenotype (Supplemental Figure 1). tmf is single-flowered, and this flower often develops leaf-like sepals (MacAlister et al., 2012; Xu et al., 2016). Interestingly, as introduction of more mutations of TFAM genes into tmf background, sepals of the solitary flower showed more leaf characteristics (Figure 2A). These findings suggest that $T M F$ requires TFAMs to achieve the precise control of flowering transition, among which TFAM1 and TFAM3 contribute much more than TFAM2.

While the primary inflorescence of tmf is single-flowered, inflorescences that develop from side shoots are unaffected (MacAlister et al., 2012), suggesting the existence of redundant factors. We then examined the inflorescences from side shoots of various higher-order mutants combined by tmf and tfams. Quantification of flower number per inflorescence from side shoots showed no significant difference between tmf, tmf tfam 1, tmf tfam 2, tmf tfam 1/2 mutants and wild type plants (Figure 2C and 2D), however, tmf tfam 1 and $t m f$ tfam $1 / 2$ displayed leaf-like sepal at the first flower on the side shoot inflorescences (Figure 2C). Notably, the side shoot inflorescences of tmf tfam3 double mutant almost always produce only two flowers, and most of the inflorescences showed vegetative reversion and branching (Figure 2C and 2D). Most remarkably, approximately 74\% of side shoot inflorescences from tmf tfam 1/3 are single-flowered with extremely leaf-like sepals (Figure 2C and 2D), which never appears in tfam 1/2/3 triple mutants (Figure 1D and 1F). Interestingly, the side shoot inflorescences of tmf tfam 1/2/3 are mostly undistinguished from tmf tfam 1/3, suggesting nonessential role of TFAM2 in this process (Figure 2C-2G). Together, these results suggest that $T M F, T F A M 1$ and TFAM3 indispensably work together in flower production 
on both primary and side shoots.

\section{TMF and TFAMs synergistically repress shoot apical meristem maturation}

To explore the developmental basis for the flowering and inflorescence defects in various single and high-order mutants, we dissected and compared the shoot apical meristems at reproductive stages. Shoot apical meristems of the tfam single and higher-mutants are indistinguishable at the transitional meristem (TM) stage (Figure 3A). However, the maturation rate of the SAM, indicated by the number of leaf primordium produced before vegetative meristems transitioning into floral meristems, varied in different mutants. Neither tfam 1 nor tfam 2 showed modified maturation rate, however, tfam 3 , tfam $1 / 2$ and tfam $2 / 3$ transitioned faster than wild type by about one leaf primordium (Figure $3 \mathrm{~A}$ and $3 \mathrm{~B}$ ). tfam $1 / 3$ and tfam $1 / 2 / 3$ exhibited the fastest maturation rate, producing about two leaf primordium fewer than wild type for floral transition (Figure 3A and 3B). The faster maturation gave rise to the early-flowering phenotypes in those mutants. The inflorescence complexity can be reflected by the number of AIM initiated at young inflorescence stage (Xu et al., 2016). tfam 1/2 shows slightly slower initiation of the AIMs, however, tfam 1/3, tfam $2 / 3$ and tfam 1/2/3 initiated remarkably fewer AIMs than wild type (Figure 3C). The precocious termination of the AIM initiation interpreted the simplified inflorescences of these tfam mutants. When examining the young inflorescence stages of various combinations of tmf and tfam mutants, one can clearly observe extremely simplified inflorescences featured by single flowers with leaf-like sepals (Figure 3D). The progressive enhancement of flowering and inflorescence defects were reflected by the number of leaf primordium production and the size of leaf-like sepals (Figure 3D and 3E). Taken together, these results indicated that TMF and TFAMs synergistically prevent precocious maturation of shoot apical meristems to ensure appropriate flowering transition and inflorescence complexity.

\section{TFAM proteins form biomolecular condensates in the nucleus of tomato cells}

The synergistic interactions and overlapped functions prompt us to investigate if TMF and TFAM proteins share conserved properties. We analyzed the protein primary structure and found that all 
three TFAMs share highly conserved ALOG domain with a zinc-ribbon motif inserted in the middle region, which is a putative DNA-binding domain derived from the XerC/D-like recombinases. They also harbor a classic nuclear localization signal (NLS) in the C-terminus (Figure 4A). Several ALOG family proteins, including TMF, have been reported to have transcriptional activity (Huang et al., 2021; Iyer and Aravind, 2012; MacAlister et al., 2012; Yoshida et al., 2013). Transcriptional activity assays in yeast showed that TFAM2 and TFAM3 displayed significantly transcriptional auto-activation in the yeast-two hybrid systems, however, no auto-activation was detected for TFAM1, probably due to its unique DNA-binding preferences (Figure 4B). To explore the protein behavior of TFAMs in living tomato cells, we expressed TFAM-GFP fusion proteins in tomato protoplast. Confocal imaging showed that all three TFAM proteins exclusively localized in the nucleus as TMF did (Figure 4C). Strikingly, the GFP signals in the nucleus show high heterogeneity that marks an aggregate or condensate state, mimicking the punctate localization pattern of TMF (Figure 4D) (Huang et al., 2021). Given the fact that TMF undergoes liquid-liquid phase separation to form transcriptional condensates in the nucleus (Huang et al., 2021), the heterogenous condensation of TFAM proteins in the nucleus is likely due to protein phase separation.

\section{TFAM proteins undergo phase separation in vitro}

Further analysis of the TFAM proteins revealed that, like TMF, all three TFAMs have prion-like intrinsically disorder regions (IDRs) that are usually considered as a signature for protein phase separation (Figure 5A). We then recombinantly expressed and purified the TFAM-GFP fusion proteins from E. coli (Supplemental Figure 2A). We used the purified proteins to perform an in vitro phase separation assay that can generate a phase diagram by systematically changing protein and salt concentrations to assess the conditions that promote condensate formation (Huang et al., 2021). Interestingly, while all three TFAM proteins underwent phase separation, they showed some varying properties. Like TMF, TFAM3 readily phase-separated into droplets with a relatively regular spherical shape, however, TFAM1 and TFAM2 formed more irregular filamentous 
assemblies, these filaments and droplets are stable during the period of observation (Figure 5B5D). The phase diagram showed a progressive increase of the density and size of the condensates formed by phase-separated TFAMs as the protein concentration improves when the salt concentration is constant (Figure 5B-5D). In contrast, the condensate abundance decreased with the increase of salt concentration when the protein concentration is constant, indicating they are sensitive to salt and protein concentrations (Figure 5B-5D). In particular, the TFAM3 proteins started to form visible spherical droplets at a concentration of $1 \mu \mathrm{M}$ in a buffer with $150 \mathrm{mM} \mathrm{NaCl}$ (a physiologically relevant salt concentration), and the droplets rapidly fused together to form large droplet clusters as protein concentration increases (Figure 5D). It seems that the phase separation capacity of TFAM2 is weaker than that of TFAM1 and TFAM3 since it is more sensitive to salt.

We then captured the fusion process of the condensates using time-lapse microscopy. The results show that the condensates formed by all three TFAM proteins can rapidly fuse by necking and relaxation to form a larger one on intersection of two droplets (Figure 5E and Supplemental Video 1-3), suggesting their dynamic property. To validate this, we performed fluorescence recovery after photobleaching (FRAP) analysis to bleach the centers of large droplets and monitored recovery. The bleached pots started to recover after several seconds, and eventually reached around $20 \%$ to $50 \%$ recovery of the originally detected signal intensity after several minutes (Figure 5F-5K and Supplemental Video 4-6). Together, our findings demonstrated that all three TFAM proteins undergo phase separation in vitro and they show varying phase separation property when existed individually.

\section{TMF selectively recruits TFAMs into phase separated condensates}

Given that TMF synergistically acts together with three TFAMs and the four proteins share protein phase separation property, together with the fact that TMF interacts with TFAM1 and TFAM2, we hypothesized that TMF interacts with three TFAMs to form a protein complex that enables formation of a "family condensate". To test this, we took advantage of the bimolecular fluorescence complementation (BiFC) assay, by which we can simultaneously detect protein- 
protein interactions and analyze phase-separated condensates in living tomato cells. We performed the pairwise interaction tests between TMF and three TFAMs. The results showed that four proteins interacted with each other in the nucleus, supporting the formation of a protein complex. Image analysis of heterogeneity of the fluorescence intensity and quantification of the cells with condensates indicated that almost all the combinations of interacting pairs induce formation of biological condensates, except the combination of TFAM1 and TFAM2, whose interaction displays homogenous fluorescence signals (Figure 6A, 6B and Supplemental Figure 3). To validate the interactions in vitro and monitor the phase separation behavior during interactions without disturbance from other potentially interacting partners, we recombinantly expressed and purified mCherry-TMF (red fluorescence) and GFP-TFAM (green fluorescence) proteins to perform crossmixing phase separation reactions (Zhou et al., 2020). Apparently, TMF can coexist with itself to form the perfectly merged droplets (Figure 6C). However, it can largely but not fully merge with TFAM1 and TFAM2 droplets (Figure 6C). Surprisingly, TMF shows the most remarkable coincidence with TFAM3 in the same droplets, almost identical to the degree of TMF with itself (Figure 6C), suggesting their tight interactions and cognate property of protein multivalence. In contrast, TMF rarely recruited TFAM11 into its droplets, indicating the selectivity of the recruitment and coexistence during phase separation (Figure 6C). Given the expression of TFAM11 gradually decreases from early vegetative meristem stage to transitional meristem stage, but dramatically increases at floral meristem stage, apparently opposite to that of TMF, the TFAM11 protein might associate with TMF at very early vegetative stages in an unintimate way, and the association was then competed by three other TFAMs due to their closer cognate property of IDR derived multivalences to TMF. Once entering into floral meristem stage, TMF disappears, but TFAM11 up-regulates to take turns to act. Taken together, TMF directly interacts with TFAM1, 2 and 3 and selectively recruits them into phase-separated condensates.

\section{The ALOG transcriptional condensates repress $\boldsymbol{A} N$ expression to synchronize flowering}

We recently reported that TMF forms transcriptional condensates to directly target floral identity 
gene $A N$ to repress its expression in the meristems before flowering transition (Huang et al., 2021). To test if TMF acts together with TFAM1/2/3 to target $A N$, we micro-dissected transitional meristems from WT, tfam 1/2/3, tmf and tmf tfam 1/2 plants for qRT-PCR analysis (Figure 7A left). The results showed that $A N$ expression was precociously activated in tfam $1 / 2 / 3$ comparing to WT (Figure 7A middle). As previously reported and shown here, $A N$ prematurely and dramatically upregulated in $t m f$ and the effect significantly enhanced in tmf tfam 1/2 triple mutant (Figure 7A right), indicating that TMF and TFAM1/2/3 synergistically repress $A N$ expression in the shoot apical meristems at the stages before flowering transition. To validate if the transcriptional repression is direct, we performed a series of transcriptional activity assays using the betaglucuronidase (GUS)-luciferase (LUC) dual reporter system in tobacco leaves, where promoter sequence of $A N$ was fused with GUS to serve as reporter and various combinations of co-expressed TMF and TFAMs served as effectors (Huang et al., 2021) (Figure 7B). The assays showed that coexpression of three TFAMs significantly improved TMF's repression on the transcription of $A N$ (Figure 7C). These results demonstrated that TMF interacts with TFAM1/2/3 to form a transcriptional complex that directly represses $A N$ expression.

The aforementioned molecular evidences were then confirmed by extensive genetic analysis. As the anantha (an) homozygous mutant repeatedly over-proliferates axillary inflorescence meristems but never forms normal flowers (Lippman et al., 2008), we then crossed anantha heterozygous mutant with single and high-order mutants of tfam and tmf. By screening progeny from $\mathrm{F}_{2}$ plants, we obtained tfam1 an, tfam 2 an, tfam 3 an, tfam 1/2 an and tmf tfam 1/2 an mutant. The inflorescences of these mutants were indistinguishable from anantha mutant, suggesting that anantha mutant is completely epistatic to tmf and tfam mutants (Figure 7D). Together, these results demonstrate that the transcriptional condensates formed by four paralogous proteins of ALOG family precisely control meristem maturation by directly repressing $A N$ expression, which ensures adequate vegetative growth for synchronizing flowering and producing compound inflorescences. 


\section{Discussion}

In this study, we uncovered a new mechanism featured by redundant paralogs within one protein family coordinate via phase separation to control a developmental program, by which tomato plants achieve precise control of shoot apical meristem maturation for flowering and compound inflorescence production. Our findings support a model wherein TMF serves a core regulator to selectively recruit its paralogous partners TFAM1, 2 and 3 into phase-separated condensates, the resulting transcriptional condensates bind to the promoter of floral identity gene $A N$ to repress it expression during vegetative meristem stages. This phase-separation based repressing program for meristem maturation enables a sufficient duration of vegetative stage for proliferating enough stem cells that ensure synchronization of flowering and production of compound inflorescences (Figure 8). To the best of our knowledge, this is the first example in plants known to elucidate how paralogous transcriptional factors within a protein family synergistically interact to undergo phase separation for stem cell fate decision. The selective-recruitment featured phase separation might represent a general mechanism that is utilized for "task assignment or coordination" among different members within a protein family.

Despite being sessile, plants have successfully propagated and robustly survived in diverse ecosystems. One key to this evolutionary success is their potent capacity to adapt to local environments (Paaby and Testa, 2018). Biological robustness, usually achieved by the presence of partially redundant parts that result from gene duplication, is an inherent ability of plants that allows them to survive and reproduce in a wide range of different ecosystems (Diss et al., 2014; de Jong and Leyser, 2012). Functional overlap between paralogs allows them to compensate for each other's loss, as commonly revealed by aggravating genetic interactions (Diss et al., 2014). Creating a full series of mutant combinations of all the ALOG paralogs involved in a certain developmental process allows us to extensively explore the functional overlaps underlying the genetic robustness. For example, when loss of one or two TMF paralogs, tomato plants can still 
produce multiple-flowered inflorescences with a subtle early-flowering phenotype (Figure 8 and Figure 1D-1F). In this situation, while plants produce fewer fruits and seeds for propagation, their population can still survive. However, once loss of all four functional overlapped ALOG genes simultaneously, tomato plants flower extremely early after producing only two true leaves and develop a single-flowered inflorescence with severe floral organ defects, leading to failure of setting fruits and seeds for propagation (Figure 8 and Figure 2A-2D). Our study discovered that plants adopt a selective-recruitment phase separation mechanism to coordinate the functional overlapped paralogs to achieve biological robustness.

The paralogous proteins within a family often share one or more highly conserved domains that define the protein family, but they often have some regions that are highly diverged. The intrinsically disorder region represents a type of highly-diverged protein sequence. IDRs are featured by their lack of stable secondary or tertiary structure and are rapidly evolving at the primary sequence level. Approximately $40 \%$ of all proteins in eukaryotic organisms are either entirely disordered or contain sizeable regions that are disordered (Ward et al., 2004; Zarin et al., 2017). The variance of IDRs among paralogous proteins is likely due to spontaneous mutation occurring during gene duplication (Zarin et al., 2017), which endows the paralogs with different capacity for phase separation and thus varied cognate recognition for functional compensation involved in an essential biological process. IDR driven protein phase separation represents a type of dynamic and flexible protein behavior that has been recently reported to implicate in acclimation responses to cellular $\mathrm{pH}$ levels, heat and oxidative stress (Franzmann et al., 2018; Kroschwald et al., 2018; Riback et al., 2017; Yang et al., 2019). The biological condensates formed by functionally associated and phase-separated paralogous proteins might serve as a common mechanism evolved in multicellular organisms for transcriptional adaption to cellular or environmental stresses. 


\section{Methods}

\section{Plant materials and growth conditions}

The tmf, an, tfam 1, tfam 2 and tfam 3 single mutants used in this study are in tomato (Solanum lycopersicum) cultivar M82 background. The higher-order mutants for tfam1/2, tfam1/3, tfam 2/3, tfam 1/2/3, tmf tfam 1, tmf tfam 2, tmf tfam 3, tmf tfam 1/2, tmf tfam 1/3, tmf tfam 1/2/3 were produced by crossing using single mutants. The homozygotes were genotyped by digestion of PCR production amplified using the primers listed in Table S1. Seedlings were grown in growth room at $26^{\circ} \mathrm{C}$, with $45-60 \%$ relative humidity under LED (Philips Lighting IBRS) light. Greenhouse plants were grown under natural light supplemented with LED. $16 \mathrm{~h}$ light $/ 8 \mathrm{~h}$ dark photoperiod was used for seedlings and greenhouse plants.

\section{Transcriptional activity assay}

To detect the transcriptional activity of ALOG proteins, the yeast two-hybrid assay was carried out as previously described (Xu et al., 2016). Plasmids for TMF-BD, TFAM1-BD, TFAM2$B D$ were described as previously (Huang et al., 2018) and the coding sequences of TFAM3 was amplified and cloned into vector pGBKT7 (BD). These plasmids were combined with vector pGADT7 (AD) and co-transformed into AH109 yeast cells, respectively. The transformed cells were plated on the selective mediums.

Meanwhile, the GUS-LUC dual reporter system as previous described was used to perform transcription activity assays in vivo (Huang et al., 2021). The ALOG proteins fused different tags served as effctors. $p A N: G U S$ served as a reporter, and 35S:LUC served as an internal control as described previously. Co-infiltrated the plasmids of effector and reporter into N.benthamiana leaves, and harvested leaves after $60 \mathrm{~h}$. Total proteins were extracted for measuring the activity of GUS and luciferase (LUC) activity using 4-methylumbelliferyl glucuronide (Sigma) and luciferin (Promega) as substrates, respectively. The transcriptional activity was determined by the ratio of GUS/LUC. 


\section{Recombinant protein expression and purification}

To generate the constructs for recombinant protein expression, coding sequences of fusion DNA fragments for eGFP-TFAMs and mCherry-TMF were cloned into the vector pQE-80L. The plasmids were transformed into E. coli Rosetta (DE3) competent cells, and positive bacteria cultured in LB was induced by $0.5 \mathrm{mM}$ isopropyl $\beta$-D-1-thiogalactopyranoside (IPTG) for $16 \mathrm{~h}$ at $16{ }^{\circ} \mathrm{C}$. Collected cells and performed purification using Ni-NTA (GE healthcare) affinity beads as previous described. Buffer exchange and concentration for eluted proteins were performed using ultrafiltration tubes (Vivaspin turbo). Purified proteins were stored in storage buffer (50 mM Tris$\mathrm{HCl}, 200 \mathrm{mM} \mathrm{NaCl}, \mathrm{pH} 7.4)$ at $-80{ }^{\circ} \mathrm{C}$ after quick-freezing in liquid nitrogen.

\section{Phase separation assay and FRAP in vitro}

The phase separation assays were performed by dilution of purified proteins into buffer containing $50 \mathrm{mM}$ Tris- $\mathrm{HCl}(\mathrm{pH} 7.4)$ and various concentrations for $\mathrm{NaCl}$ to indicated final concentrations in figure legends. Purified proteins were centrifuged $10 \mathrm{~min}$ at 14,000g and transferred supernatants into new tubes to exclude the effects caused by precipitated proteins. To generate phase diagram, diluted phase-separated protein solution was incubated for $15 \mathrm{~min}$ at room temperature in a 384well plate. To perform droplet interaction assay for TMF and TFAMs in vitro, purified proteins dissolved in buffer containing $50 \mathrm{mM}$ Tris- $\mathrm{HCl}(\mathrm{pH} 7.4), 25 \mathrm{mM} \mathrm{NaCl}$ as indicated in figure legends were thoroughly mixed and incubated for $15 \mathrm{~min}$ at room temperature in a 384-well plate. Images for droplets and filaments were taken using confocal microscopy (Nikon A1R + ) equipped with $\times 20, \times 40$ and $\times 100$ oil objectives. Fluorescence was excited at 488 and detected at 500-540 for GFP, excited at $543 \mathrm{~nm}$ and detected at 595-635 $\mathrm{nm}$ for mCherry.

\section{Subcellular localization and $\mathrm{BiFC}$ assays in tomato Protoplasts}

To investigate the subcellular localization of TFAM proteins, we generated the constructs. The coding sequences of TFAM1 and TFAM3 were amplified and separately cloned into transient expression vector to generate 35S:eGFP-TFAM1, 35S:TFAM3-eGFP, and cloned into pSCYNE (SCN) and pSCYCE (SCC) to generate TFAM3-N-CFP (TFAM3-SCN) and TFAM3-C-CFP (TFAM3-SCC) BiFC assay. Plasmids for 35S:TMF-eGFP, 35S:TFAM2-eGFP, TMF-SCC, TMF- 
SCN, TFAM1-SCC, TFAM1-SCN, TFAM2-SCC and TFAM2-SCN were described as previously (Huang et al., 2018). Plasmids were transfected into protoplasts isolated from tomato cotyledons as previous described. Fluorescent signals detection was performed using confocal microscopy (Leica SP5) with $\times 20, \times 40$ objectives.

\section{Author Contributions}

C.X. designed the research; X.H., and N.X. performed most of the experiments and analysed the data; Y.X., L.T., Y.Z., and Y.Y. provided helps in genotyping and plasmid constructions; C.X. wrote the paper with input from X.H..

\section{Acknowledgments}

We thank Z. B. Lippman (Cold Spring Harbor Laboratory) for providing mutant seeds. This study was supported by the Major Research Plan of National Natural Science Foundation of China (31991183), Key Research Program of Frontier Sciences of the Chinese Academy of Science (ZDBS-LY-SM021), the Strategic Priority Research Program of Chinese Academy of Sciences (XDA24030503) to C.X.; China Postdoctoral Science Founding (2018M641516) and a Natural Science Founding (31900174) to X.H.. No conflict interest declared.

\section{References}

Alberti, S., and Hyman, A.A. (2021). Biomolecular condensates at the nexus of cellular stress, protein aggregation disease and ageing. Nature Reviews Molecular Cell Biology 22, 196-213.

Banani, S.F., Lee, H.O., Hyman, A.A., and Rosen, M.K. (2017). Biomolecular condensates: organizers of cellular biochemistry. Nature Reviews Molecular Cell Biology 18, 285-298.

Barton, M.K. (2010). Twenty years on: The inner workings of the shoot apical meristem, a developmental dynamo. Developmental Biology 341, 95-113.

Benlloch, R., Berbel, A., Serrano-Mislata, A., and Madueño, F. (2007). Floral initiation and inflorescence architecture: A comparative view. Annals of Botany 100, 659-676. 
Boeynaems, S., Alberti, S., Fawzi, N.L., Mittag, T., Polymenidou, M., Rousseau, F., Schymkowitz, J., Shorter, J., Wolozin, B., Van Den Bosch, L., et al. (2018). Protein Phase Separation: A New Phase in Cell Biology. Trends in Cell Biology 28, 420-435.

Chen, F., Zhou, Q., Wu, L., Li, F., Liu, B., Zhang, S., Zhang, J., Bao, M., and Liu, G. (2019). Genome-wide identification and characterization of the ALOG gene family in Petunia. BMC Plant Biology 19, 600.

Cho, E., and Zambryski, P.C. (2011). ORGAN BOUNDARY1 defines a gene expressed at the junction between the shoot apical meristem and lateral organs. Proceedings of the National Academy of Sciences 108, 2154-2159.

Diss, G., Ascencio, D., DeLuna, A., and Landry, C.R. (2014). Molecular mechanisms of paralogous compensation and the robustness of cellular networks. Journal of Experimental Zoology Part B: Molecular and Developmental Evolution 322, 488-499.

Emenecker, R.J., Holehouse, A.S., and Strader, L.C. (2021). Biological Phase Separation and Biomolecular Condensates in Plants. Annual Review of Plant Biology 72, annurev-arplant081720-015238.

Fang, X., Wang, L., Ishikawa, R., Li, Y., Fiedler, M., Liu, F., Calder, G., Rowan, B., Weigel, D., Li, P., et al. (2019). Arabidopsis FLL2 promotes liquid-liquid phase separation of polyadenylation complexes. Nature 569, 265-269.

Franzmann, T.M., Jahnel, M., Pozniakovsky, A., Mahamid, J., Holehouse, A.S., Nüske, E., Richter, D., Baumeister, W., Grill, S.W., Pappu, R. V., et al. (2018). Phase separation of a yeast prion protein promotes cellular fitness. Science 359, eaao5654.

González-Suárez, P., Walker, C.H., and Bennett, T. (2020). Bloom and bust: understanding the nature and regulation of the end of flowering. Current Opinion in Plant Biology.

Hendelman, A., Zebell, S., Rodriguez-Leal, D., Dukler, N., Robitaille, G., Wu, X., Kostyun, J., Tal, L., Wang, P., Bartlett, M.E., et al. (2021). Conserved pleiotropy of an ancient plant homeobox gene uncovered by cis-regulatory dissection. Cell.

Hepworth, S.R., Klenz, J.E., and Haughn, G.W. (2006). UFO in the Arabidopsis inflorescence apex is required for floral-meristem identity and bract suppression. Planta 223, 769-778.

Huang, X., Tang, L., Yu, Y., Dalrymple, J., Lippman, Z.B., and Xu, C. (2018). Control of flowering and inflorescence architecture in tomato by synergistic interactions between ALOG transcription factors. Journal of Genetics and Genomics 45, 557-560.

Huang, X., Chen, S., Li, W., Tang, L., Zhang, Y., Yang, N., Zou, Y., Zhai, X., Xiao, N., Liu, W., et al. (2021). ROS regulated reversible protein phase separation synchronizes plant flowering. Nature Chemical Biology.

Iyer, L.M., and Aravind, L. (2012). ALOG domains: provenance of plant homeotic and 
developmental regulators from the DNA-binding domain of a novel class of DIRS1-type retroposons. Biology Direct 7, 39.

de Jong, M., and Leyser, O. (2012). Developmental Plasticity in Plants. Cold Spring Harbor Symposia on Quantitative Biology 77, 63-73.

Kroschwald, S., Munder, M.C., Maharana, S., Franzmann, T.M., Richter, D., Ruer, M., Hyman, A.A., and Alberti, S. (2018). Different Material States of Pub1 Condensates Define Distinct Modes of Stress Adaptation and Recovery. Cell Reports 23, 3327-3339.

Lei, Y., Su, S., He, L., Hu, X., and Luo, D. (2019). A member of the ALOG gene family has a novel role in regulating nodulation in Lotus japonicus. Journal of Integrative Plant Biology 61, 463-477.

Lippman, Z.B., Cohen, O., Alvarez, J.P., Abu-Abied, M., Pekker, I., Paran, I., Eshed, Y., and Zamir, D. (2008). The making of a compound inflorescence in tomato and related nightshades. PLoS Biology 6, 2424-2435.

MacAlister, C.A., Park, S.J., Jiang, K., Marcel, F., Bendahmane, A., Izkovich, Y., Eshed, Y., and Lippman, Z.B. (2012). Synchronization of the flowering transition by the tomato terminating flower gene. Nature Genetics 44, 1393-1398.

Nan, W., Shi, S., Jeewani, D.C., Quan, L., Shi, X., and Wang, Z. (2018). Genome-Wide Identification and Characterization of wALOG Family Genes Involved in Branch Meristem Development of Branching Head Wheat. Genes 9, 510.

Naramoto, S., Jones, V.A.S., Trozzi, N., Sato, M., Toyooka, K., Shimamura, M., Ishida, S., Nishitani, K., Ishizaki, K., Nishihama, R., et al. (2019). A conserved regulatory mechanism mediates the convergent evolution of plant shoot lateral organs. PLOS Biology 17, e3000560.

Paaby, A.B., and Testa, N.D. (2018). Developmental Plasticity and Evolution. In Evolutionary Developmental Biology, (Cham: Springer International Publishing), pp. 1-14.

Park, S.J., Jiang, K., Schatz, M.C., and Lippman, Z.B. (2012). Rate of meristem maturation determines inflorescence architecture in tomato. Proceedings of the National Academy of Sciences 109, 639-644.

Park, S.J., Eshed, Y., and Lippman, Z.B. (2014). Meristem maturation and inflorescence architecture-lessons from the Solanaceae. Current Opinion in Plant Biology 17, 70-71.

Rebocho, A.B., Bliek, M., Kusters, E., Castel, R., Procissi, A., Roobeek, I., Souer, E., and Koes, R. (2008). Role of EVERGREEN in the Development of the Cymose Petunia Inflorescence. Developmental Cell 15, 437-447.

Riback, J.A., Katanski, C.D., Kear-Scott, J.L., Pilipenko, E. V., Rojek, A.E., Sosnick, T.R., and Drummond, D.A. (2017). Stress-Triggered Phase Separation Is an Adaptive, Evolutionarily Tuned Response. Cell 168, 1028-1040.e19. 
Rickett, H.W. (1944). The classification of inflorescences. The Botanical Review 10, 187-231.

Sato, D.-S., Ohmori, Y., Nagashima, H., Toriba, T., and Hirano, H.-Y. (2014). A role for TRIANGULAR HULL1 in fine-tuning spikelet morphogenesis in rice. Genes \& Genetic Systems 89, 61-69.

Souer, E., Rebocho, A.B., Bliek, M., Kusters, E., de Bruin, R.A.M., and Koes, R. (2008).

Patterning of inflorescences and flowers by the F-Box protein DOUBLE TOP and the LEAFY homolog ABERRANT LEAF AND FLOWER of petunia. The Plant Cell 20, 2033-2048.

Strzyz, P. (2019). Phase separation tunes signal transduction. Nature Reviews Molecular Cell Biology 20, 263-263.

Takeda, S., Hanano, K., Kariya, A., Shimizu, S., Zhao, L., Matsui, M., Tasaka, M., and Aida, M. (2011). CUP-SHAPED COTYLEDON1 transcription factor activates the expression of LSH4 and LSH3, two members of the ALOG gene family, in shoot organ boundary cells. Plant Journal $66,1066-1077$.

Teo, Z.W.N., Song, S., Wang, Y.-Q., Liu, J., and Yu, H. (2014). New insights into the regulation of inflorescence architecture. Trends in Plant Science 19, 158-165.

Ward, J.J., Sodhi, J.S., McGuffin, L.J., Buxton, B.F., and Jones, D.T. (2004). Prediction and Functional Analysis of Native Disorder in Proteins from the Three Kingdoms of Life. Journal of Molecular Biology.

Weberling, F. (1989). Morphology of flowers and inflorescences. (Cambridge: Cambridge Univeristy Press).

Wu, Q., Xu, F., and Jackson, D. (2018). All together now, a magical mystery tour of the maize shoot meristem. Current Opinion in Plant Biology 45, 26-35.

Wu, X., Cai, Q., Feng, Z., and Zhang, M. (2020). Liquid-Liquid Phase Separation in Neuronal Development and Synaptic Signaling. Developmental Cell 55, 18-29.

Xu, C., Park, S.J., Van Eck, J., and Lippman, Z.B. (2016). Control of inflorescence architecture in tomato by BTB/POZ transcriptional regulators. Genes \& Development 30, 2048-2061.

Yang, Y.S., Kato, M., Wu, X., Litsios, A., Sutter, B.M., Wang, Y., Hsu, C.H., Wood, N.E., Lemoff, A., Mirzaei, H., et al. (2019). Yeast Ataxin-2 Forms an Intracellular Condensate Required for the Inhibition of TORC1 Signaling during Respiratory Growth. Cell 177, 697710.e17.

Yoshida, A., Suzaki, T., Tanaka, W., and Hirano, H.-Y. (2009). The homeotic gene long sterile lemma (G1) specifies sterile lemma identity in the rice spikelet. Proceedings of the National Academy of Sciences of the United States of America 106, 20103-20108.

Yoshida, A., Sasao, M., Yasuno, N., Takagi, K., Daimon, Y., Chen, R., Yamazaki, R., Tokunaga, 
H., Kitaguchi, Y., Sato, Y., et al. (2013). TAWAWA1, a regulator of rice inflorescence architecture, functions through the suppression of meristem phase transition. Proceedings of the National Academy of Sciences of the United States of America 110, 767-772.

Zarin, T., Tsai, C.N., Nguyen Ba, A.N., and Moses, A.M. (2017). Selection maintains signaling function of a highly diverged intrinsically disordered region. Proceedings of the National Academy of Sciences 114, E1450-E1459.

Zhang, Y., Li, Z., Chen, N., Huang, Y., and Huang, S. (2020). Phase separation of Arabidopsis EMB1579 controls transcription, mRNA splicing, and development. PLOS Biology 18, e3000782.

Zhao, L., Nakazawa, M., Takase, T., Manabe, K., Kobayashi, M., Seki, M., Shinozaki, K., and Matsui, M. (2004). Overexpression of LSH1, a member of an uncharacterised gene family, causes enhanced light regulation of seedling development. Plant Journal 37, 694-706.

Zhou, M., Li, W., Li, J., Xie, L., Wu, R., Wang, L., Fu, S., Su, W., Hu, J., Wang, J., et al. (2020). Phase-separated condensate-aided enrichment of biomolecular interactions for high-throughput drug screening in test tubes. Journal of Biological Chemistry 295, 11420-11434.

Zhu, Y., and Wagner, D. (2020). Plant inflorescence architecture: The formation, activity, and fate of axillary meristems. Cold Spring Harbor Perspectives in Biology 12. 
Figures and figure legends

A

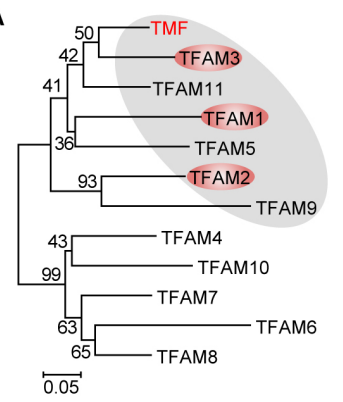

D

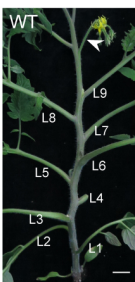

tram $1 / 27$ Reversion $25 \%$

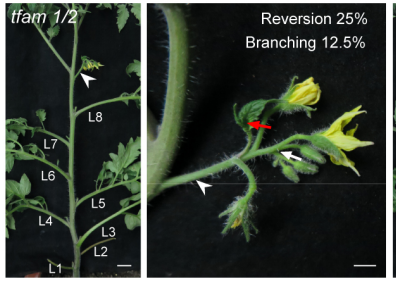

E

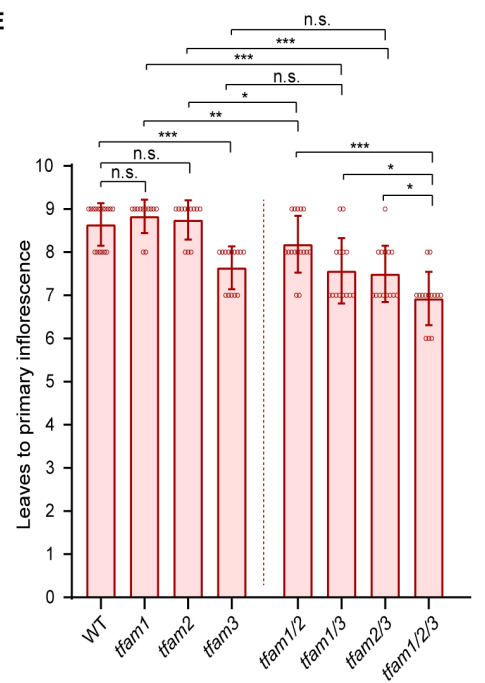

B RPKM

C
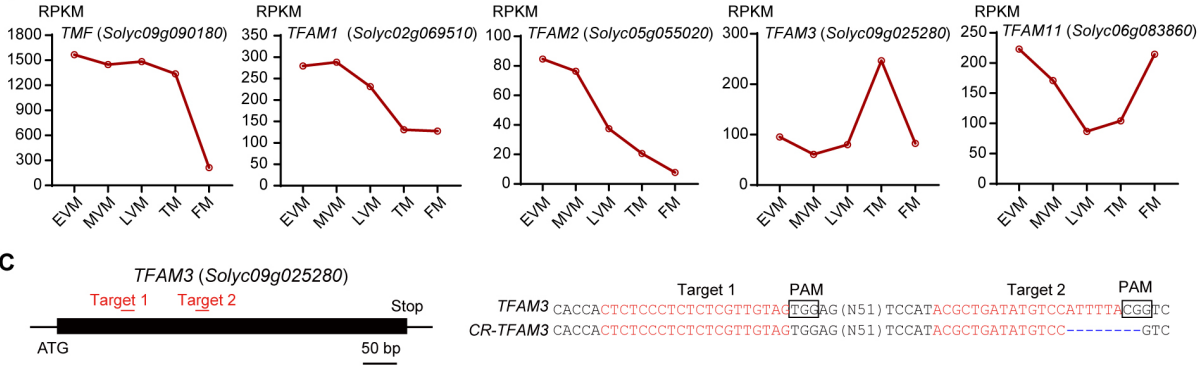

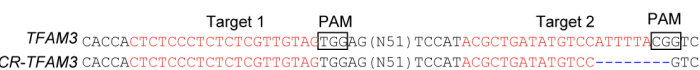

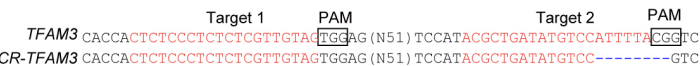
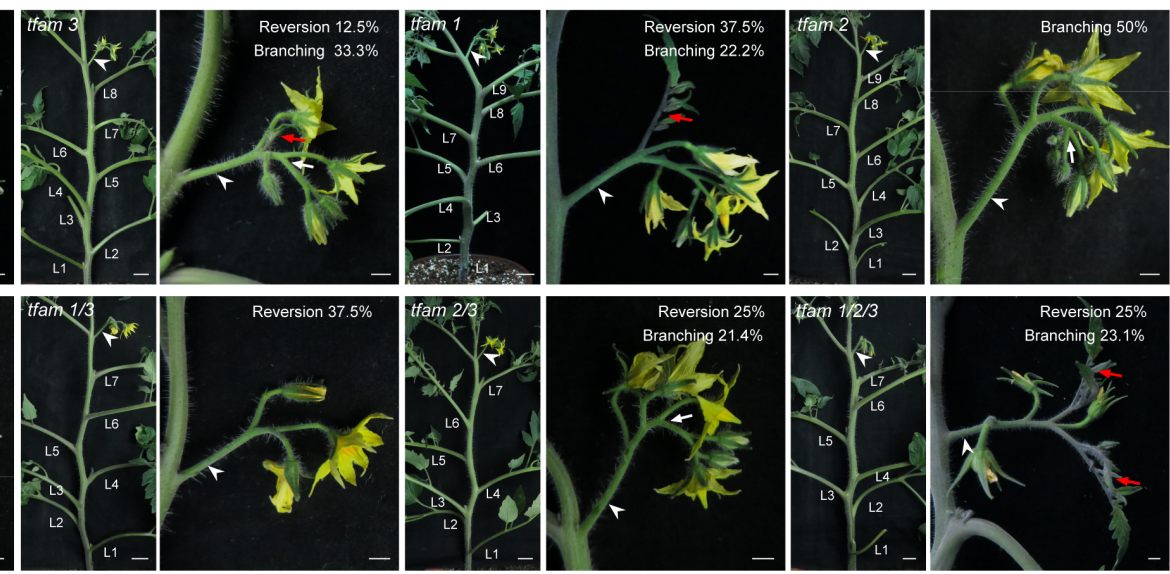

$\mathbf{F}$
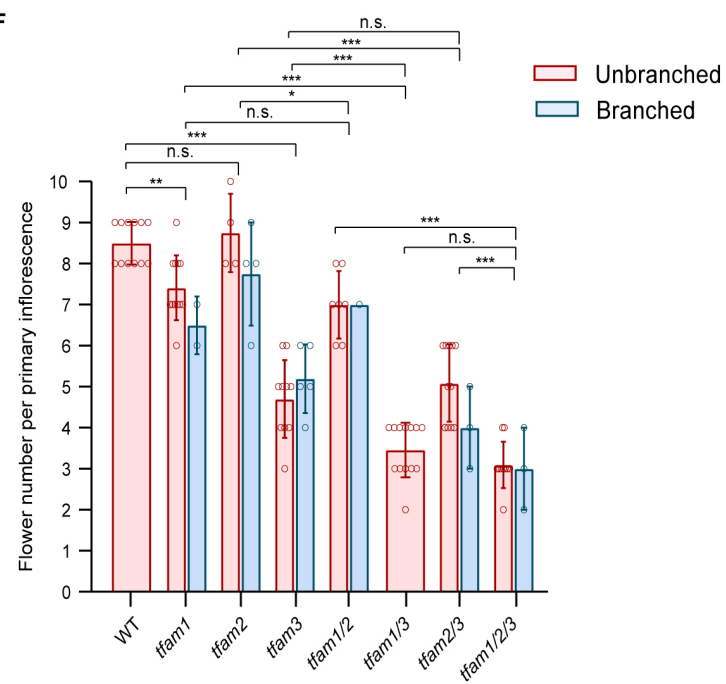

Figure 1. Identification of TFAM3 as a new regulator of flowering transition

(A) Phylogenetic tree showing the evolutionary relationship of ALOG family members in tomato. 
Red oval marked genes showing similar expression dynamics to $T M F$ in meristems.

(B) Expression dynamics for normalized read counts of TMF, TFAM1, TFAM2, TFAM3, and TFAM11 at indicated stages during meristem maturation.

(C) Schematic (left) indicating two sgRNAs (red lines) and allelic information (right) for TFAM3.

(D) Representative shoot and typical primary inflorescence from WT and tfam single, double and triple mutants. White arrowheads indicate inflorescences, red arrows indicate vegetative reversions, white arrows indicate branching events on inflorescences. L, leaf. Scale bars, $2 \mathrm{~cm}$ for plants; 0.5 $\mathrm{cm}$ for inflorescences.

(E and F) Statistics of flowering time (E) and flower number per inflorescence (F) for WT and tfam single, double and triple mutants. The flower numbers were quantified from branched and unbranched inflorescences separately. Data are means $\pm \operatorname{SD}(n=17,12,12,14,16,14,14,14$, for $\mathbf{E} ; \mathrm{n}=12,9,8,15,8,13,14,13$ for $\mathbf{F},{ }^{*} P<0.05,{ }^{* *} P<0.01,{ }^{* * *} P<0.001$, Student $t$-test). 
A
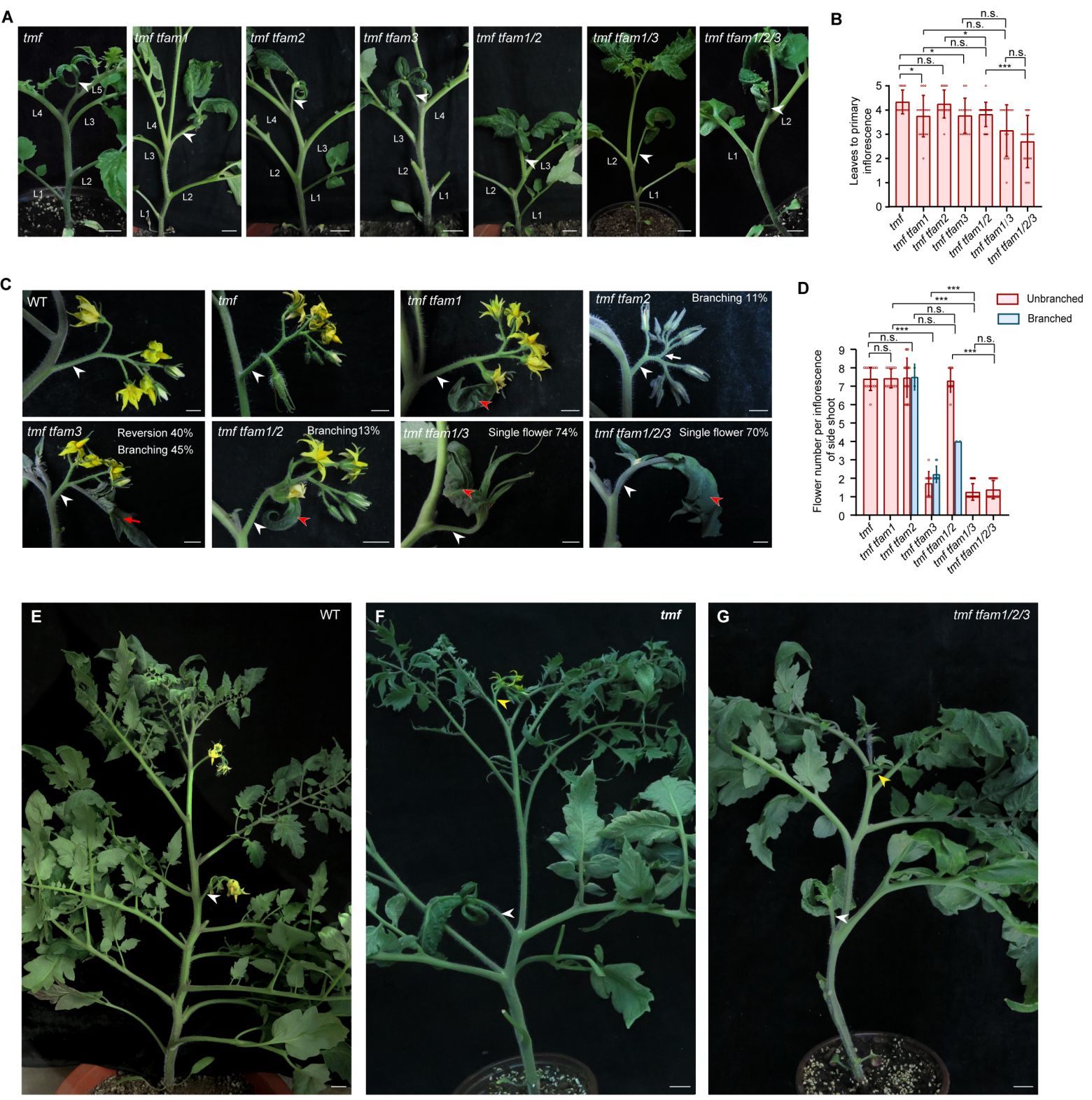

Figure 2. Genetic interactions between TMF and TFAM genes

(A and B) Representative shoots with primary inflorescence (A) and quantification of flowering time (B) for tmf single mutant and higher-order mutants of tmf and tfams. White arrowheads indicate single-flowered primary inflorescences. Data are means $\pm \mathrm{SD}(\mathrm{n}=12,16,16,13,22,13$, $20,{ }^{*} P<0.05,{ }^{* *} P<0.01,{ }^{* * *} P<0.001$, Student $t$-test). L, leaf. Scale bars, $2 \mathrm{~cm}$. 
(C and D) Images of inflorescence (C) and quantification of flower number per inflorescence (D) from side shoots of various mutant combinations. Red arrowheads indicate leaf-like sepal, and white arrowheads indicate inflorescences. Data are means $\pm \mathrm{SD}(\mathrm{n}=15,14,19,20,15,22,25$, ${ }^{* * *} P<0.001$, Student $t$-test). Scale bars, $1 \mathrm{~cm}$.

(E-G) Representative shoot with two successive inflorescences for WT (E), tmf single mutant (F) and tmf tfam 1/2/3 quadruple mutant (G). White arrowheads and yellow arrowheads indicate primary inflorescences and side-shoot inflorescences, respectively. Scale bars, $2 \mathrm{~cm}$. 

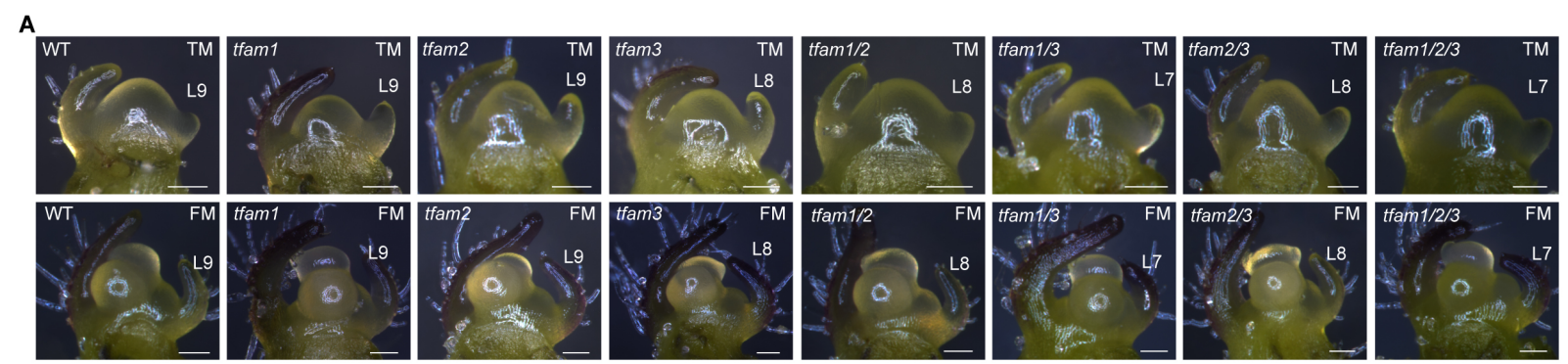

B

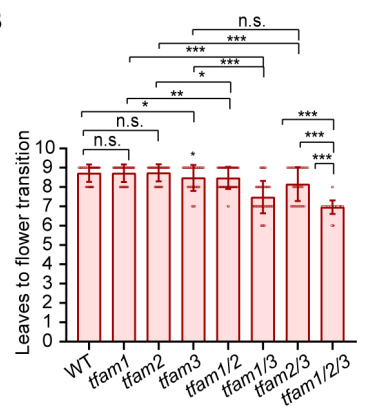

C
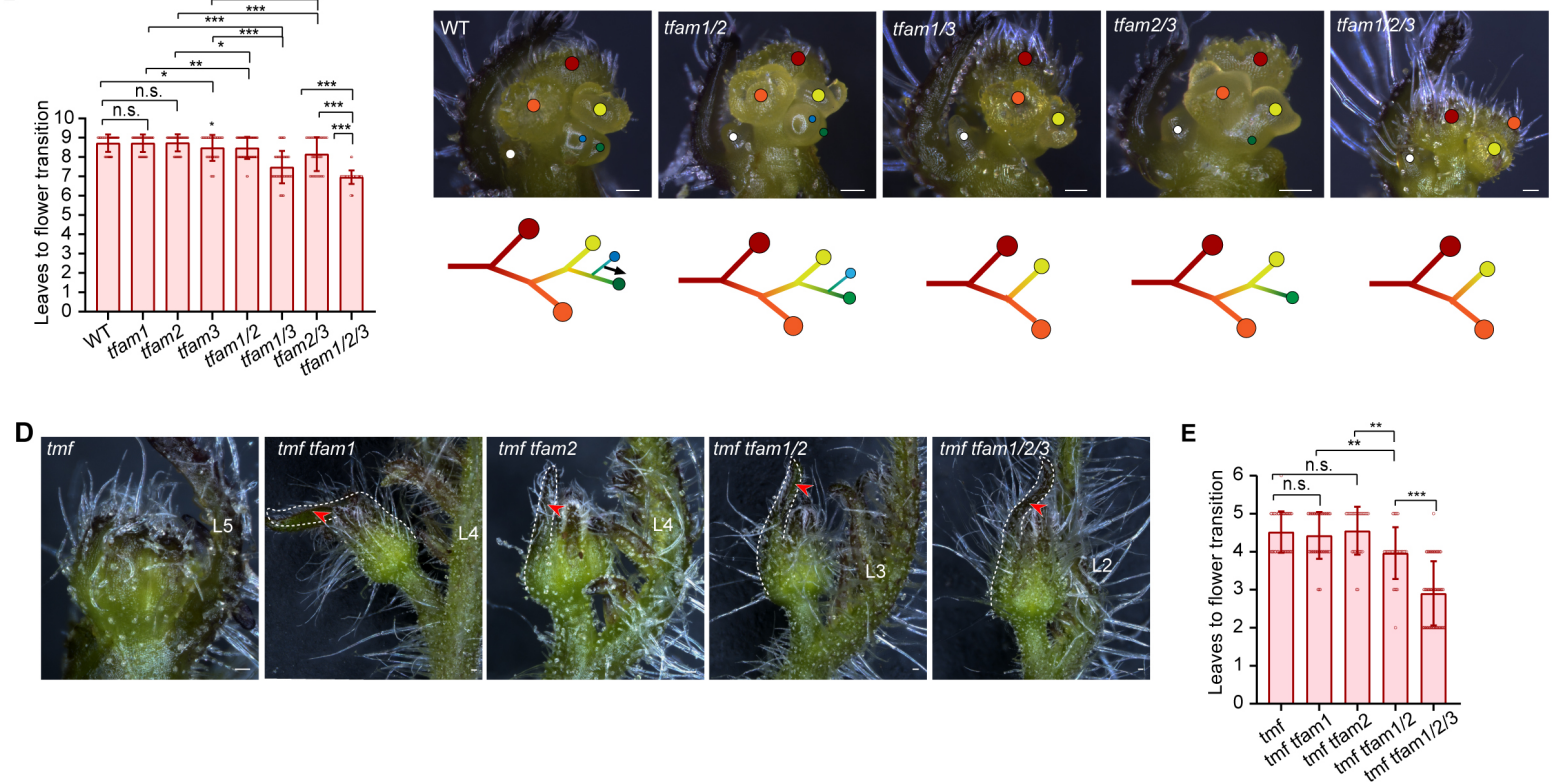

Figure 3. Developmental basis of shoot apical meristem maturation in tmf and tfam mutants.

(A and B) Stereoscope images of meristems (A) and quantification data of leave primordium production for flower transition (B) from WT and tfam single, double and triple mutants. Data are means \pm SD $\left(\mathrm{n}=69,46,46,42,59,54,39,73,{ }^{*} P<0.05,{ }^{* *} P<0.01,{ }^{* * *} P<0.001\right.$, Student $t$-test $)$. Scale bars, $100 \mu \mathrm{m}$. L, leaf.

(C) Young inflorescences (upper) and diagrams (bottom) of WT and tfam mutants. Colored dots indicate terminated FM (red, orange and yellow dots) and initiated SIM (blue and green dots). White dots indicate the first SYM. The black arrow indicates continued SIM reiteration. Scale bars, $100 \mu \mathrm{m}$. 
(D and E) Stereoscope images of floral meristem (D) and quantification of leaf production for flower transition (E) from tmf and tfam mutants. Data are means $\pm \mathrm{SD}(\mathrm{n}=53,42,29,29,62$, ${ }^{* *} P<0.01,{ }^{* * *} P<0.001$, Student $t$-test). Red arrowheads indicating the leaf sepal at floral meristem stage. Scale bars, $100 \mu \mathrm{m}$. L, leaf. 
A

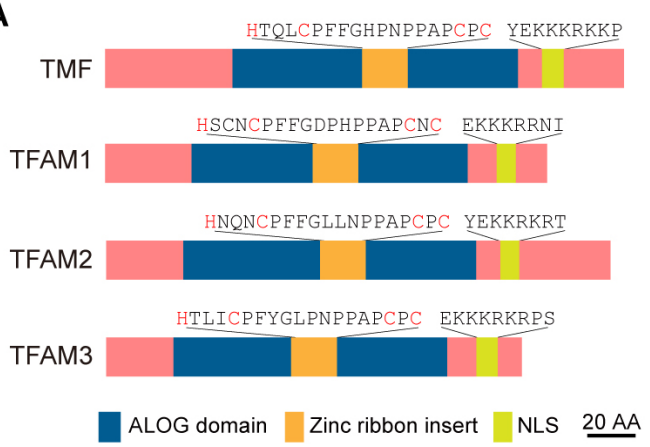

B

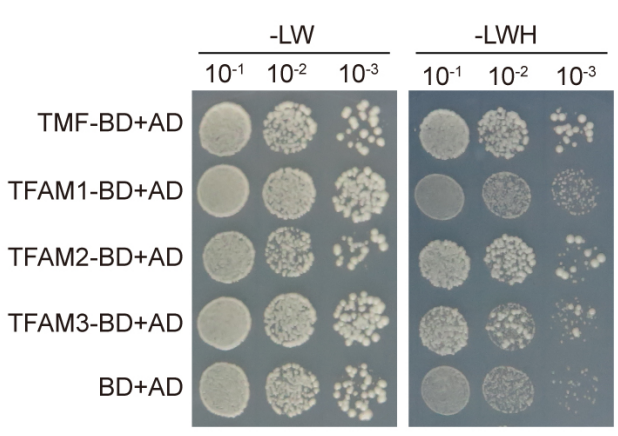

C

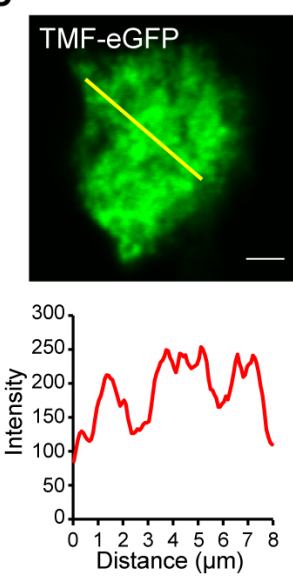

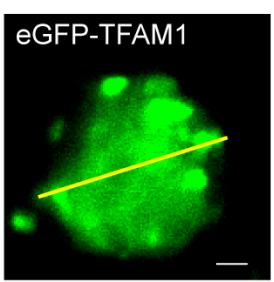

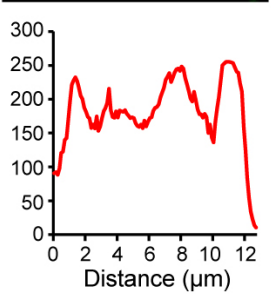

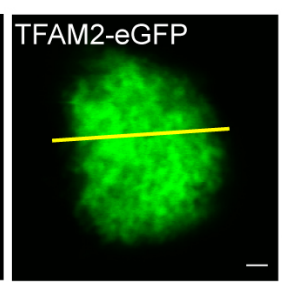

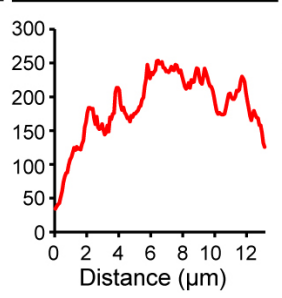

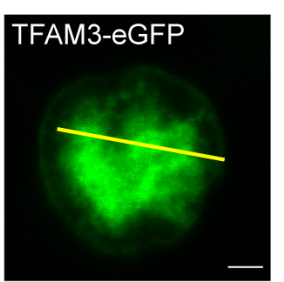

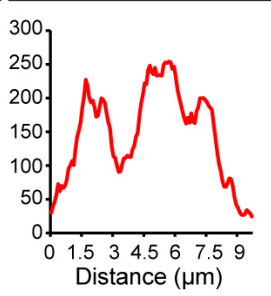

D

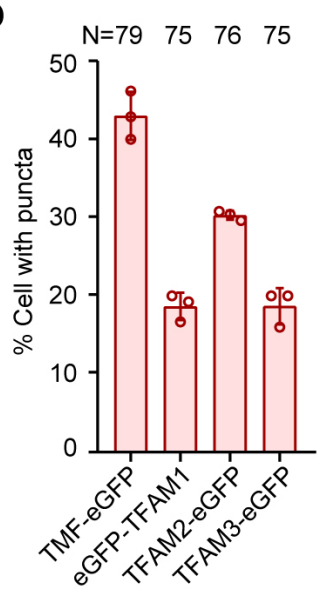

\section{Figure 4. Protein behavior of TFAM proteins in yeast and tomato cells}

(A) Schematics illustrating protein domains for TMF and TFAM proteins.

(B) Yeast two-hybrid showing the auto-activation effects of TFAM proteins. Yeast cells were screened on the medium: SD-LW (-Leu/-Trp) and SD-LWH (-Leu/-Trp/-His). AD, activation domain; BD, DNA-binding domain.

(C) Subcellular localization of TFAMs showing condensates in the nucleus of tomato cells (upper) and fluorescence intensity of indicated yellow lines (bottom). Scale bars, $2 \mu \mathrm{m}$.

(D) Quantitative data showing the percentage of cells with condensates for GFP fusion proteins of TMF, TFAM1, TFAM2 and TFAM3 in the nucleus. Data are presented as three biological 
replicates $\pm \mathrm{SD}(\mathrm{n}=79,75,76,75)$.

A

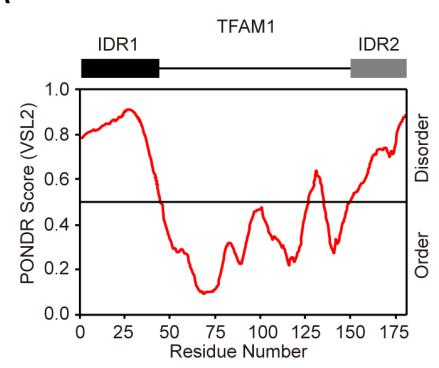

B

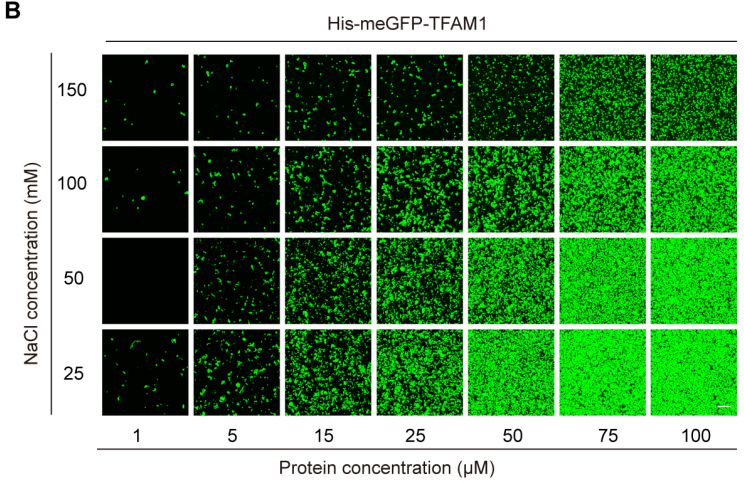

D

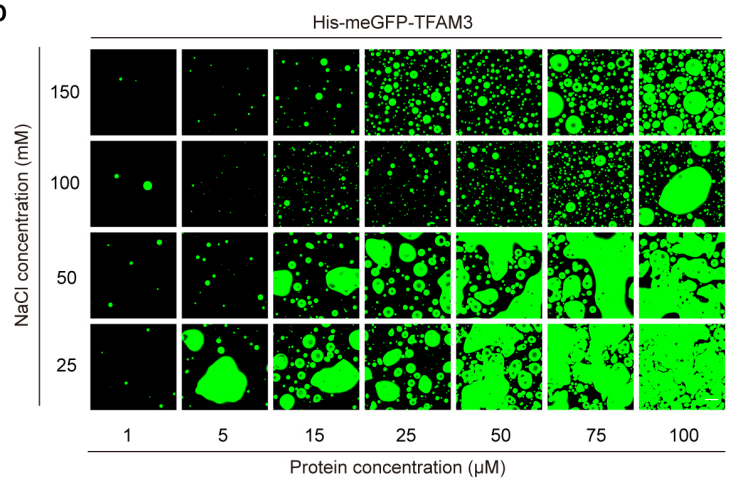

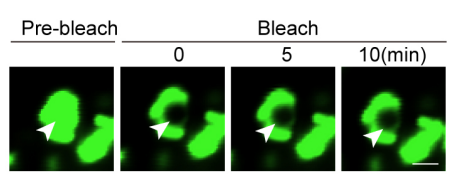

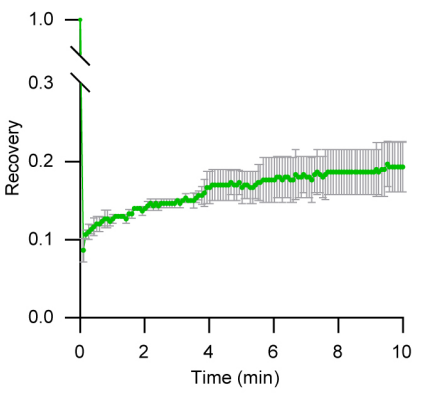

H Pre-bleach
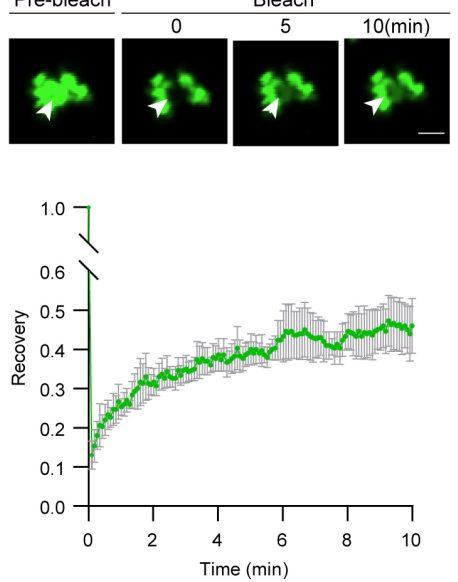

C

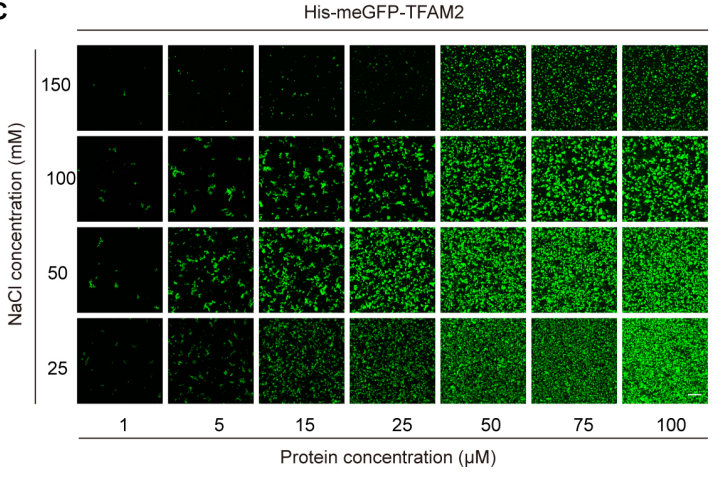

$\mathrm{E}$

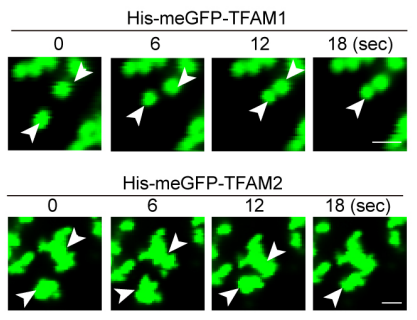

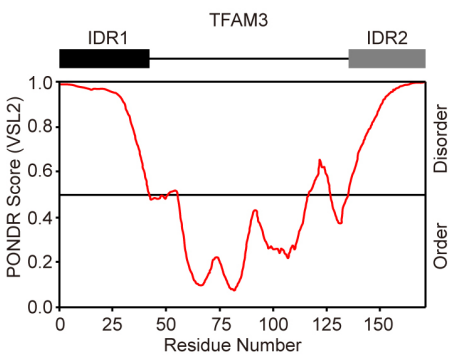

His-meGFP-TFAM2

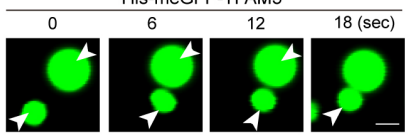

$J$

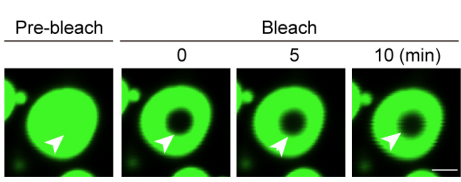

K

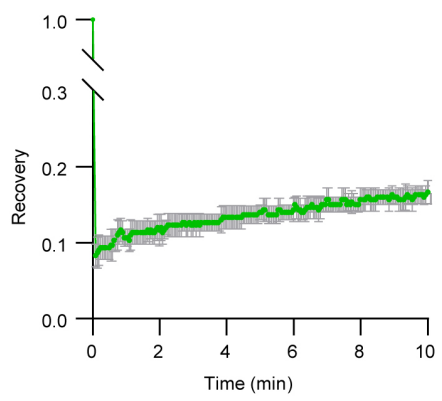




\section{Figure 5. TFAM proteins undergo phase separation in vitro}

(A) Graphs showing IDRs of TFAM proteins.

(B-D) Phase separation of GFP-TFAM1 (B), GFP-TFAM2 (C) and GFP-TFAM3 (D) under the different combinations of indicated concentrations for $\mathrm{NaCl}$ and proteins. Scale bars, $20 \mu \mathrm{m}$. Three independent experiments with similar results were performed.

(E) Representative images from three independent fusion events showing the liquidity of GFPTFAM1 (upper), GFP-TFAM2 (middle) and GFP-TFAM3 (bottom) during phase separated droplets formation. Protein concentration, $15 \mu \mathrm{M}$; NaCl concentration, $25 \mathrm{mM}$. Scale bars, $2 \mu \mathrm{m}$. (F-K) Representative images and quantification data of FRAP analysis for GFP-TFAM1 (F and G), GFP-TFAM2 (H and I) and GFP-TFAM3 (J and K). Data are means of three independent FRAP events. Scale bars, $2 \mu \mathrm{m}$. 

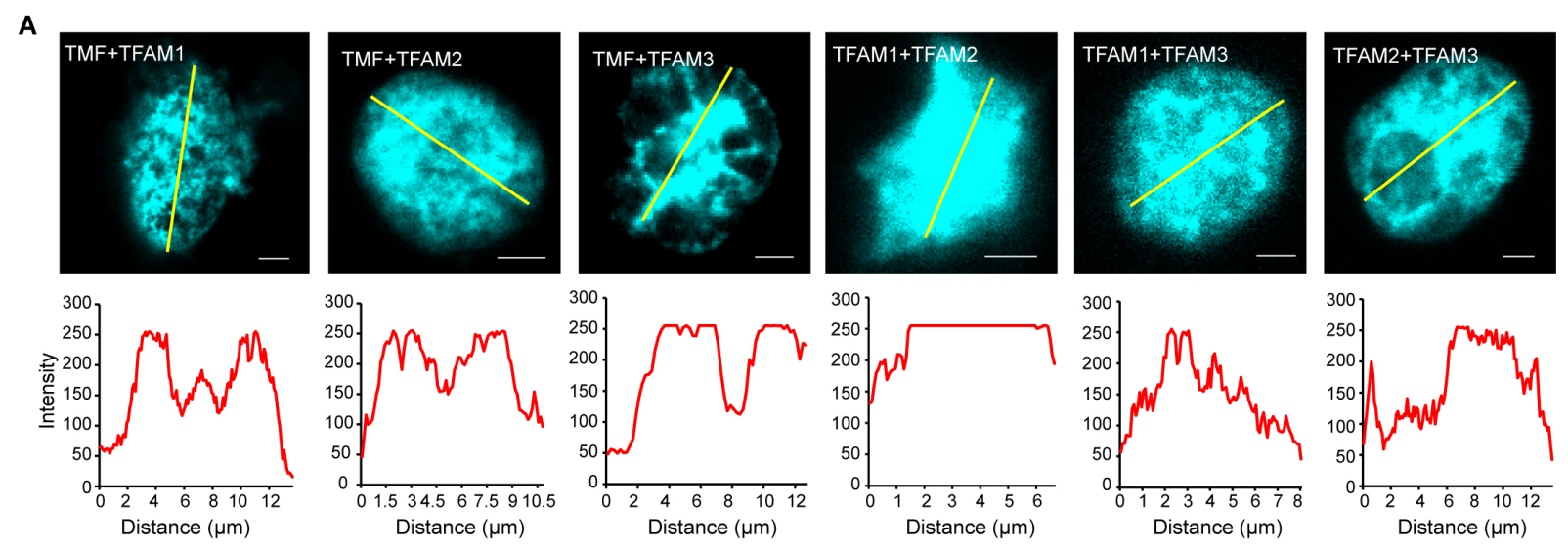

B

C
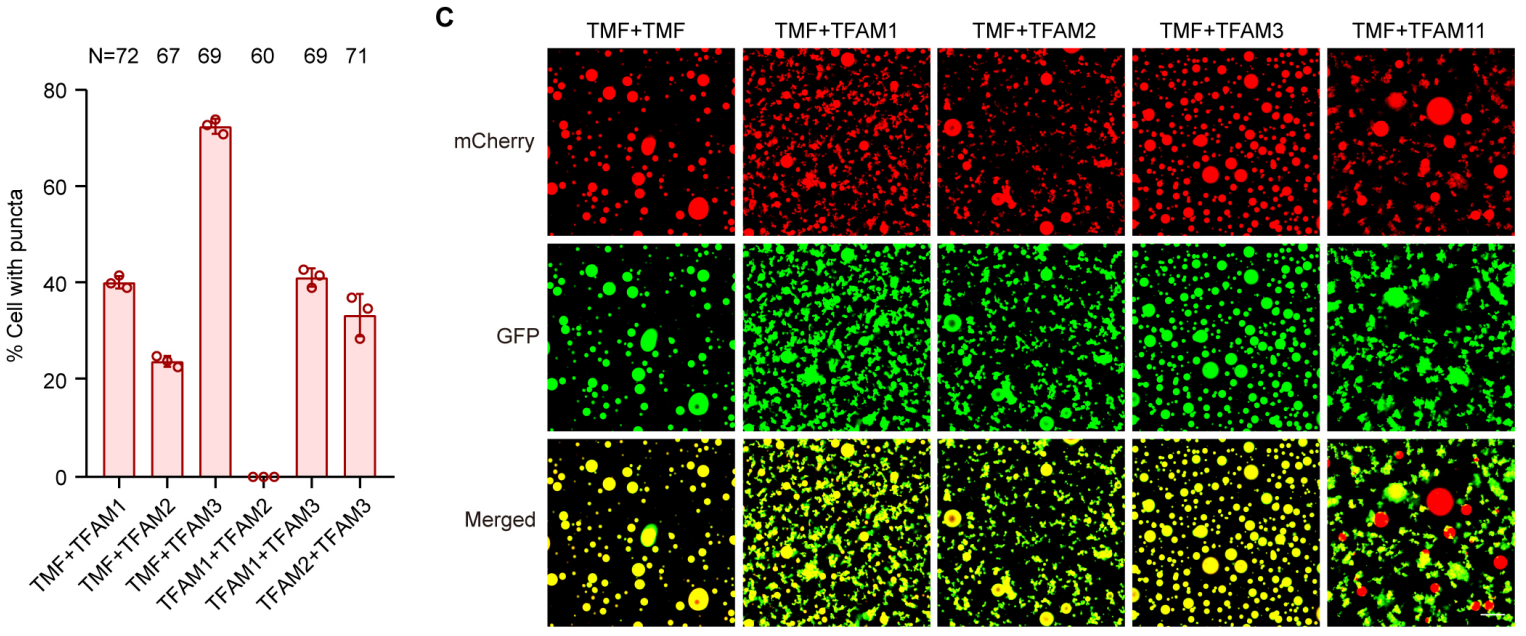

Figure 6. TMF interacts with TFAM proteins to form transcriptional condensates

(A) Representative images (upper) showing the interactions between ALOG proteins in BiFC assays. The fluorescence intensity of indicated yellow lines (bottom) showing the heterogenous condensates formed from interactions between TFAM proteins. Scale bars, $2 \mu \mathrm{m}$.

(B) Quantitative data showing the percentage of cells with condensates formed by interactions between TFAM proteins in nuclei. Data are presented as three biological replicates \pm SD ( $n=72,67$, $69,60,69,71)$.

(C) Cross-mixing phase separation reactions using recombinantly expressed mCherry-TMF fusion proteins and GFP fusion proteins of TFAMs. Protein concentration, $15 \mu \mathrm{M}$; $\mathrm{NaCl}$ concentration, 
$25 \mathrm{mM}$. Scale bar, $20 \mu \mathrm{m}$.

A
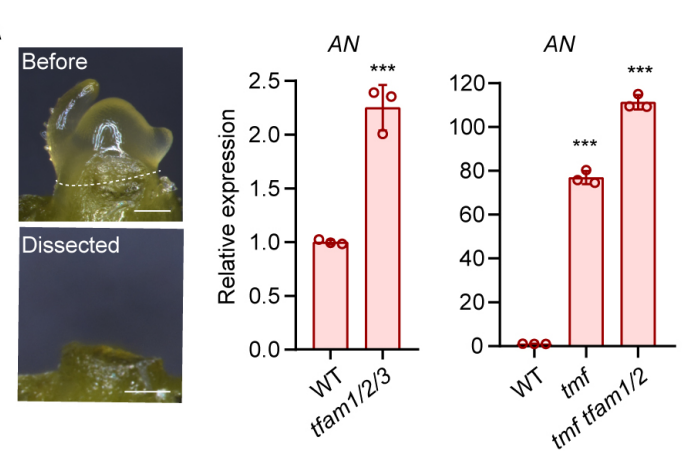

B

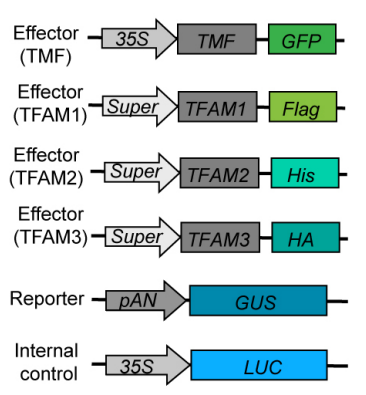

C

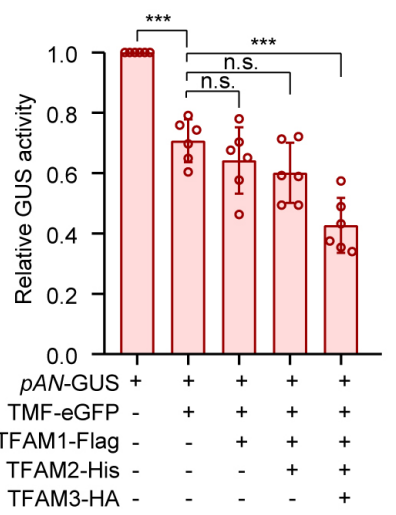

D
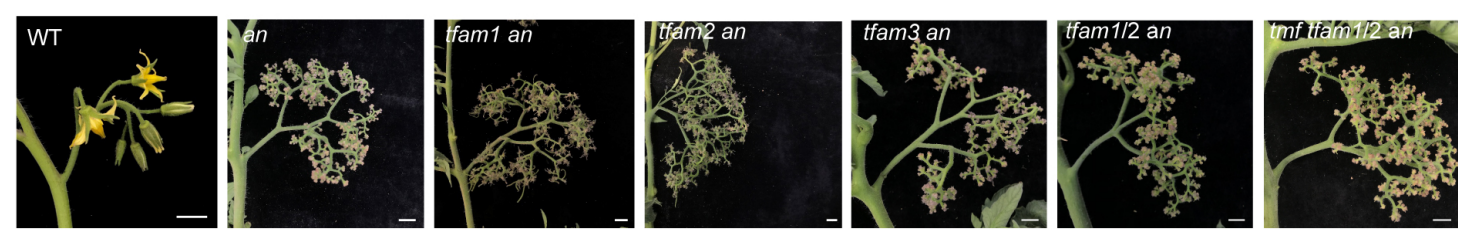

Figure 7. The ALOG transcriptional condensates repress $A N$ expression to synchronize

\section{flowering}

(A) Stereoscope images (left) of the micro-dissected transitional meristem for real-time PCR (right). White dashed line indicates the dissection position. The relative expression of $A N$ was normalized to WT using UBIQUITIN $(U B I)$ as an internal control. Data are presented as three repelicates $\pm \mathrm{SD}\left(\mathrm{n}=3,{ }^{* * *} P<0.001\right.$, Student $t$-test). Three independent experiments with similar results were carried out. Scale bars, $100 \mu \mathrm{m}$.

(B) Schematics of constructs used to analyze transcriptional activity.

(C) Transcriptional repression of $A N$ by transcriptional condensates formed from TMF and TFAM proteins. The ratio of GUS to LUC indicates relative transcriptional activity. LUC served as an internal control. Data are presented as six biological replicates from two independent experiments. Data are means $\pm \mathrm{SD}\left(\mathrm{n}=6,{ }^{* * *} P<0.001\right.$, Student $t$-test $)$.

(D) Representative images for primary inflorescences of WT, an and higher-order mutants of an and tfams. Scale bars, $1 \mathrm{~cm}$. 

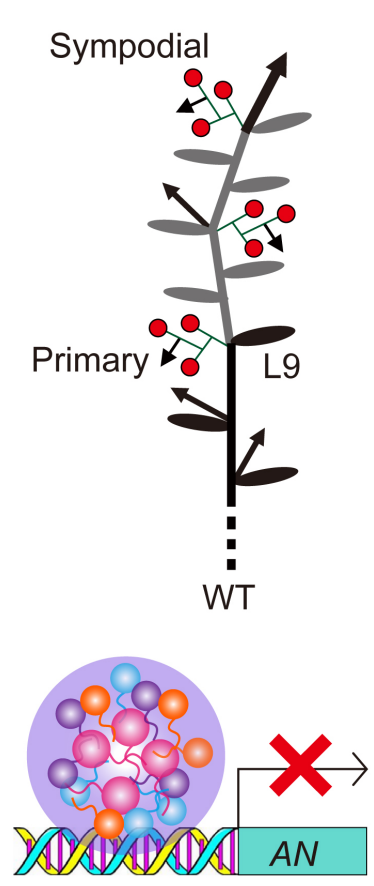
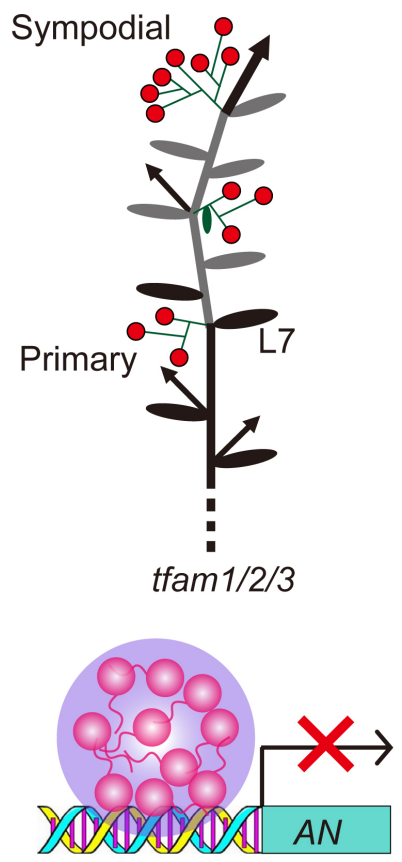
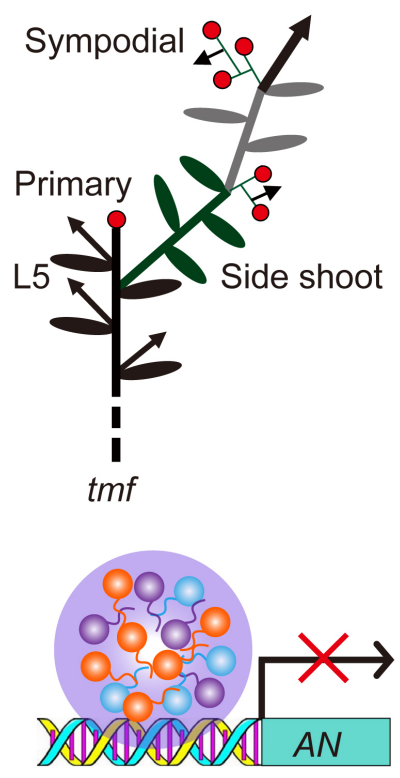

O TMF

On TFAM1

○ TFAM2

On TFAM3

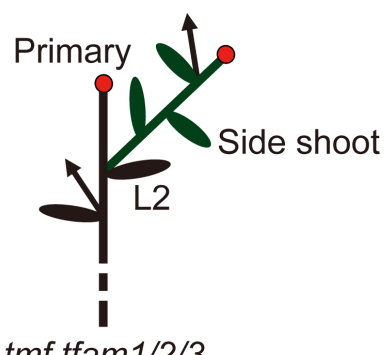

tmf tfam $1 / 2 / 3$

Transcriptional repression capacity

Figure 8. Working model for transcriptional condensates formed by phase-seperated ALOG family proteins in synchronizing flowering and promoting inflorescence complexity. 


\section{Supplemental information}

Transcriptional condensates formed by phase-separated ALOG family proteins control flowering and inflorescence architecture in tomato

Xiaozhen Huang, Nan Xiao, Yue Xie, Lingli Tang, Yueqin Zhang, Yuan Yu, and Cao Xu

Supplemental Video 1. Fusion for two phase-separated GFP-TFAM1 filaments.

Supplemental Video 2. Fusion for two phase-separated GFP-TFAM2 filaments.

Supplemental Video 3. Fusion for two phase-separated GFP-TFAM3 droplts.

Supplemental Video 4. FRAP analysis of phase-separated GFP-TFAM1 in vitro.

Supplemental Video 5. FRAP analysis of phase-separated GFP-TFAM2 in vitro.

Supplemental Video 6. FRAP analysis of phase-separated GFP-TFAM3 in vitro. 


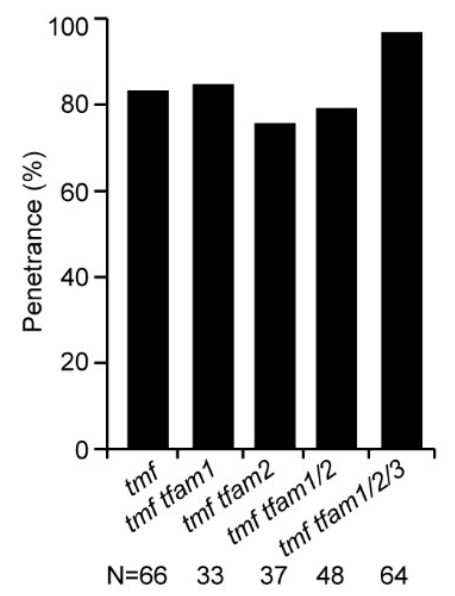

Figure S1. Quantification of penetrance for $\operatorname{tm} f$ single mutant and higher-order mutants of tmf and tfams. 


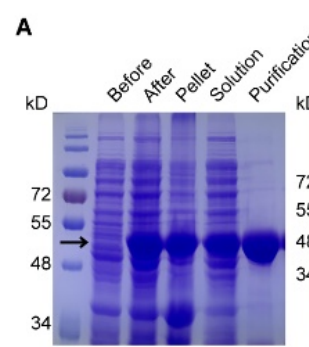

His-eGFP-TFAM1

B

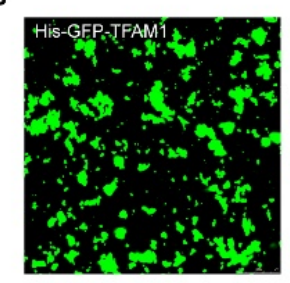

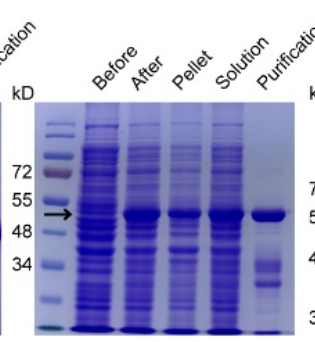

His-eGFP-TFAM2

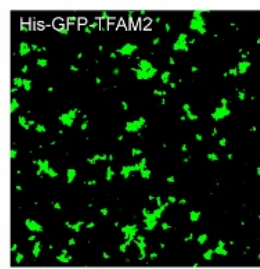

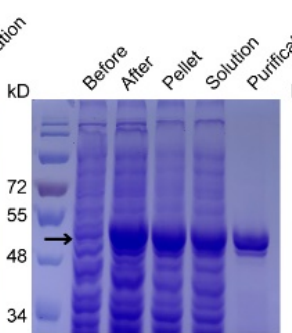

His-eGFP-TFAM3

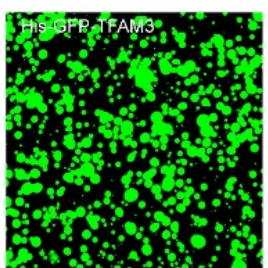

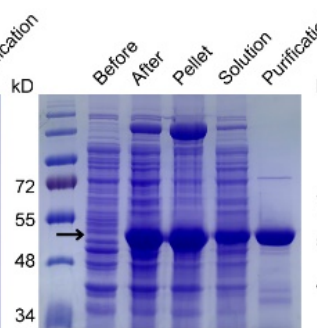

His-eGFP-TFAM11

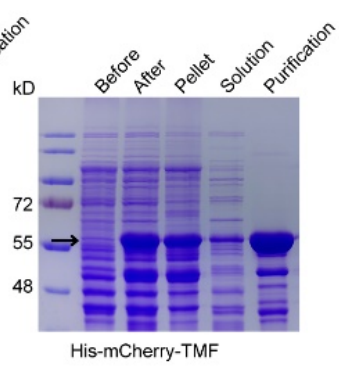

His-mCherry-TMF

Figure S2. Protein purification for phase separation analysis in vitro.

(A) SDS -PAGE gels showing the inducing and purification for His-eGFP or His-mCherry fused proteins. The black arrows indicate target bands of proteins, respectively.

(B) Images of phase separation for His-eGFP-TFAMs and His-mCherry-TMF used in this study.

Proteins concentration, $15 \mu \mathrm{M}$. NaCl concentration, $25 \mathrm{mM}$. Scale bar, $20 \mu \mathrm{m}$. 

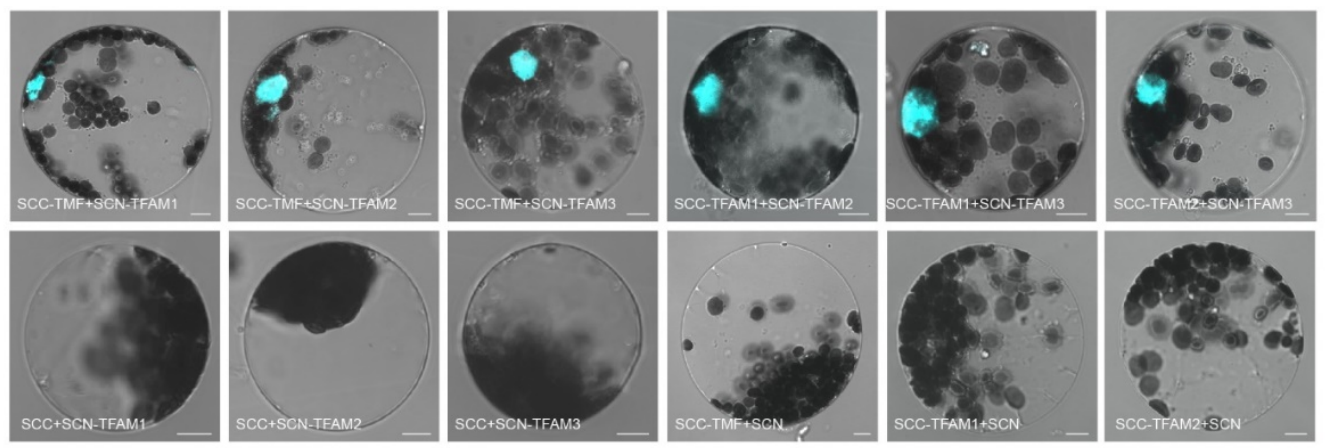

Figure S3. BiFC assays showing the interactions between TMF and TFAMs in nucleus of tomato protoplast. Scale bars, $5 \mu \mathrm{m}$. 
Table S1. Primer list used in this study

\begin{tabular}{|c|c|}
\hline Primer name & Primer sequence \\
\hline \multicolumn{2}{|c|}{ Cloning of constructs for recombinant protein expression } \\
\hline$P Q E-T F A M 1-\mathrm{F}$ & $\begin{array}{l}\text { GGACGAGCTGTACAAGGGCCGGCCTGGAGGTGGAGGTGGAGCTATGTT } \\
\text { AGACGTATATA }\end{array}$ \\
\hline$P Q E-T F A M 1-\mathrm{R}$ & CCAAGCTCAGCTAATTAAGCTTCTAATCACAATTATTAATAGTACTAC \\
\hline$P Q E-T F A M 2-\mathrm{F}$ & GGAGGTGGAGGTGGAGCT AAGCTT ATGGATTTTGTCACAGCACAAGG \\
\hline$P Q E-T F A M 2-\mathrm{R}$ & GCTCAGCTAATTAAGCTT TCAATTACCATTTCCAATATTTCCAT \\
\hline$P Q E-T F A M 3-\mathrm{F}$ & GGAGGTGGAGCT AAGCTT ATGGATTCCTTTGTAGAAGTAGA \\
\hline$P Q E-T F A M 3-\mathrm{R}$ & GCTCAGCTAATTAAGCTT TCATCCCGATTGTAATGGTGGTGG \\
\hline$P Q E-T F A M 11-\mathrm{F}$ & GGAGGTGGAGCT AAGCTT ATGGCTTCTTTTACGGAATTAGTT \\
\hline PQE-TFAM11-R & GCTCAGCTAATTAAGCTT TCATAACTCAAATGGTAATGCACC \\
\hline$P Q E-m C h e r r y-T M F-F$ & $\begin{array}{l}\text { GCATGGACGAGCTGTACAAGGGCCGGCCTGGAGGTGGAGGTGGAGCTA } \\
\text { AGCTTATGGAACACAACCAAGAAGTGG }\end{array}$ \\
\hline$P Q E-m C h e r r y-T M F-\mathrm{R}$ & CCAGCTCAGCTAATTAAGCTTTTAGCTTGAATTTCCATTTGGTGGTGGC \\
\hline \multicolumn{2}{|c|}{ Genotyping for homozygotes } \\
\hline tfam3-geno-F & CCTCATCAAGTCGATATGAGAATC \\
\hline tfam3-geno-R & CAGGATTTCCACCATTTTCTTC \\
\hline \multicolumn{2}{|c|}{ Cloning of constructs for 35S:eGFP-TFAM1 } \\
\hline$e G F P-\mathrm{F}$ & GTCGACCTCGAGGGTACC ATGGTGAGCAAGGGCGAGGAGC \\
\hline$e G F P-\mathrm{R}$ & GGCCCCAGCGGCCGCAGCAG \\
\hline TFAM1-F & CTGCGGCCGCTGGGGCCATGTTAGACGTATATAGTAC \\
\hline TFAM1-R & TCGGGGAAATTCGAGCTC CTAATCACAATTATTAATAG \\
\hline \multicolumn{2}{|c|}{ Cloning of constructs for $\mathrm{Y} 2 \mathrm{H}$ assay } \\
\hline$B D-T F A M 3-F$ & CATGGAGGCCGAATTCATGGATTCCTTTGTAGAAGTAGAGC \\
\hline$B D-T F A M 3-R$ & GGATCCCCGGGAATTCTCATCCCGATTGTAATGGTGGTGGT \\
\hline
\end{tabular}

